\title{
Primordial black holes from modified supergravity
}

\author{
Yermek Aldabergenov ${ }^{1,2, a}$ (D) Andrea Addazi $^{3,4, b}$, Sergei V. Ketov ${ }^{5,6,7, c}$ \\ ${ }^{1}$ Department of Physics, Faculty of Science, Chulalongkorn University, Thanon Phayathai, Pathumwan, Bangkok 10330, Thailand \\ ${ }^{2}$ Institute of Experimental and Theoretical Physics, Al-Farabi Kazakh National University, 71 Al-Farabi Avenue, Almaty 050040, Kazakhstan \\ ${ }^{3}$ Center for Theoretical Physics, College of Physics, Science and Technology, Sichuan University, 610065 Chengdu, China \\ ${ }^{4}$ INFN, Sezione Roma Tor Vergata, 00133 Rome, Italy \\ ${ }^{5}$ Department of Physics, Tokyo Metropolitan University, 1-1 Minami-ohsawa, Hachioji-shi, Tokyo 192-0397, Japan \\ ${ }^{6}$ Research School of High-Energy Physics, Tomsk Polytechnic University, 2a Lenin Avenue, Tomsk 634028, Russian Federation \\ ${ }^{7}$ Kavli Institute for the Physics and Mathematics of the Universe (WPI), The University of Tokyo Institutes for Advanced Study, Kashiwa 277-8583, \\ Japan
}

Received: 14 July 2020 / Accepted: 25 September 2020 / Published online: 6 October 2020

(c) The Author(s) 2020

\begin{abstract}
The modified supergravity approach is applied to describe a formation of Primordial Black Holes (PBHs) after Starobinsky inflation. Our approach naturally leads to the two-(scalar)-field attractor-type double inflation, whose first stage is driven by scalaron and whose second stage is driven by another scalar field which belongs to a supergravity multiplet. The scalar potential and the kinetic terms are derived, the vacua are studied, and the inflationary dynamics of those two scalars is investigated. We numerically compute the power spectra and we find the ultra-slow-roll regime leading to an enhancement (peak) in the scalar power spectrum. This leads to an efficient formation of PBHs. We estimate the masses of PBHs and we find their density fraction (as part of Dark Matter). We show that our modified supergravity models are in agreement with inflationary observables, while they predict the PBH masses in a range between $10^{16}$ $\mathrm{g}$ and $10^{20} \mathrm{~g}$. In this sense, modified supergravity provides a natural top-down approach for explaining and unifying the origin of inflation and the PBHs Dark Matter.
\end{abstract}

\section{Introduction}

The prospect that Dark Matter (DM) is composed of Primordial Black Holes (PBHs) is an intriguing and highly motivated alternative to any particle physics explanations such as Weak Interacting Massive Particles (WIMPs), gravitino or axion dark matter. Indeed, such a possibility reverses the strategy for DM phenomenology: DM signals may appear in cosmological data rather than colliders, direct detection

\footnotetext{
a e-mail: yermek.a@chula.ac.th (corresponding author)

be-mail: addazi@scu.edu.cn

c e-mail: ketov@tmu.ac.jp
}

searches or indirect detection in astroparticle physics. The idea of PBHs was proposed by Zeldovich and Novikov [1], and then by Hawking [2] who realized that some primordial density fluctuations may lead to PBH seeds in the early Universe. There are several mechanisms that may catalyze the formation of PBHs: (i) gravitational instabilities induced from scalar fields [3] such as axion-like particles or multifield inflation, (ii) bubble-bubble collisions from first order phase transitions (see Refs. [4-6] for recent discussions), and (iii) formation of critical topological defects such as cosmic strings [7] and domain walls [8,9] in the early Universe.

After accretion, some PBHs may survive in the Universe today and provide candidates for (non-particle) Dark Matter (DM) [10]. More recently, PBHs attracted considerable attention in the literature, related to observational progress in lensing, cosmic rays, and Cosmic Microwave Background (CMB) radiation, see Refs. [11,12] for a review of observational constraints on PBHs and their prospects for being a fraction of or a whole DM.

On the theoretical side, PBHs are considered as a probe of very high energy physics and quantum gravity "even if they never formed" [13]. Numerous phenomenological scenarios were proposed for PBH formation and, especially, for PBH generation after inflation in the early Universe, under the assumption that PBHs contribute to DM (see e.g., Refs. [14-20] and the references therein). Indeed, the whole PBH DM case leaves only two limited windows for allowed PBH masses around either $10^{-15}$ or $10^{-12}$ of the Solar mass.

Therefore, it is of interest to study a possible theoretical origin of PBHs at a more fundamental level than General Relativity (GR) by using string theory, as a candidate for quantum gravity, and supergravity as the first step in that direction. Moreover, because of the constraints imposed by local 
supersymmetry on possible couplings, a viable description of PBHs in supergravity may lead to significant discrimination of phenomenological models of inflation and PBHs.

Due to the absence of large non-Gaussianities and isocurvature perturbations in the current observational CMB data [21], single-field inflationary models were distinguished and discriminated within the large landscape of inflation mechanisms. The Starobinsky $R^{2}$ inflation [22] seems to be favored as the best phenomenological fit. Then a required growth (by a factor of $10^{7}$ compared to the CMB amplitude) of the amplitude of fluctuations to be responsible for PBH seeds can be achieved by modifying the inflaton scalar potential with a nearly inflection point [23-25]. Details of the PBH production after single-field inflation are very much dependent upon a choice of inflaton potential. This requires a significant finetuning for PBHs as a candidate of DM. Standard (Einstein) supergravity can accommodate single-field inflationary models in the (new) minimal setup, with the only restriction to the inflaton potential as a real function squared [26-29]. ${ }^{1}$

Since there are no fundamental reasons for the absence of non-Gaussianities and iso-curvature perturbations (they just have to be below the observational limits), multi-field inflationary models were also extensively studied. The required growth of primordial fluctuations can be achieved by tachyonic instabilities, say, in the waterfall phase of hybrid inflation [31,32]. Moreover, the $\mathrm{PBH}$ production may be a generic feature of two-field inflation [33]. On the other side, multifield inflation considerably extends a number of physical degrees of freedom and possible interactions, which reduce predictive power.

Thus, supersymmetry is expected to be even more important in multi-field inflation by limiting the number of fields involved (in the minimal setup) and severely restricting their interactions.

As a guiding principle, in this paper we elaborate on a possible "supergravitational" origin of both inflation and PBHs, by using only supergravity fields and their locally supersymmetric interactions, without adding extra matter fields. Only the minimal number of the physical degrees of freedom associated with an $N=1$ full supergravity multiplet is used. In its spirit, our approach is similar to Starobinsky inflation based on gravitational interactions only (see Ref. [34] for a recent review of Starobinsky inflation in gravity and supergravity). The Starobinsky inflation is based on the modified $\left(R+\zeta R^{2}\right)$ gravity, which can be further extended to modified supergravity in the minimal setup [35-37] leading to the effective two-field double inflation. We will show that the emerging double-field inflationary model is suitable for a formation of PBH seeds after the first inflation. In this sense, Starobinsky

\footnotetext{
${ }^{1}$ See e.g., Ref. [30] for a specific example of the inflaton potential with an inflection point in supergravity.
}

supergravity naturally relates inflation with the dark matter genesis.

Our paper is organized as follows. In Sect. 2 we introduce the general modified supergravity setup and give a specific example of the bosonic terms arising in the simplest non-trivial model. In Sect. 3 we introduce the duality transformations between the modified supergravity and the standard supergravity (in Jordan and Einstein frames) in terms of the field components (of the bosonic part) and in terms of the superfields. In Sect. 4 we study the vacuum structure of our basic model and the effective inflationary dynamics of its two scalars. Section 5 is devoted to an investigation of two-field inflation in our basic model defined by keeping only the leading terms in a generic modified supergravity action. We demonstrate consistency of the basic model with $\mathrm{CMB}$ observations but also find the necessity of extreme finetuning of initial conditions for PBH generation. In Sect. 6 we extend our basic model by two subleading terms within the same modified supergravity framework, and study in detail the two modifications of our basic model, corresponding to activation of only one of the two subleading terms. We numerically compute the power spectra, and estimate PBH masses and their density fraction, in both cases. We find that our extended models are capable to simultaneously describe viable (Starobinsky-type) inflation and $\mathrm{PBH}$ production after inflation, with limited fine-tuning of the parameters, and an attractor-type behavior in one of our models. In Sect. 7, we give our conclusions and comments. Some technical details are summarized in Appendices A and B.

\section{Modified supergravity setup}

Let us consider a modified supergravity theory with the general Lagrangian (in curved superspace of the old-minimal supergravity in four spacetime dimensions, with $M_{\mathrm{Pl}}=1$ ) $[36,38]$

$\mathcal{L}=\int d^{2} \Theta 2 \mathcal{E}\left[-\frac{1}{8}\left(\overline{\mathcal{D}}^{2}-8 \mathcal{R}\right) N(\mathcal{R}, \overline{\mathcal{R}})+\mathcal{F}(\mathcal{R})\right]+$ h.c.,

which is parametrized by two arbitrary functions, a nonholomorphic real potential $N$ and a holomorphic potential $\mathcal{F}$, of the covariantly chiral scalar curvature superfield $\mathcal{R}$ of the old-minimal supergravity. ${ }^{2}$ Some relevant details about supergravity in superspace are collected in Appendix A. It should be mentioned that the master Eq. (1) goes beyond the supergravity textbooks and describes a modified supergravity because the standard (Einstein) supergravity actions are the extensions of Einstein-Hilbert term, whereas Eq. (1) is more

\footnotetext{
2 We use the standard (Wess-Bagger) notation [39] for supergravity in superspace with a few adjustments mentioned in Appendix A.
} 
general and reduces to the pure Einstein supergravity action only in the very special case of $N=0$ and $\mathcal{F}=-3 \mathcal{R}$. In other words, Eq. (1) can be considered as a generic modified supergravity extension of $\left(R+R^{2}\right)$ gravity (see below).

Let us expand the functions $N$ and $\mathcal{F}$ in Taylor series and keep only the leading terms, as our first probe of modified supergravity. Then our simplest non-trivial ansatz reads

$N=\frac{12}{M^{2}} \mathcal{R} \overline{\mathcal{R}}-\frac{\xi}{2}(\mathcal{R} \overline{\mathcal{R}})^{2}, \quad \mathcal{F}=\alpha+3 \beta \mathcal{R}$,

where we have introduced the real parameters $M$ and $\xi$, and the complex parameters $\alpha$ and $\beta$. The ansatz in Eq. (2) was already proposed in Ref. [37], and it also appeared in the dual scalar-tensor supergravity (see Sect. 3) in Ref. [40] where it was shown that the $\xi$-term is essential for curing a tachyonic instability of inflation.

After expanding the Lagrangian above in terms of the field components (see Appendix A for the definitions of the field components), we obtain the bosonic part as follows:

$$
\begin{aligned}
e^{-1} \mathcal{L}= & -\frac{1}{12}\left[3(\beta+\bar{\beta})-\frac{24}{M^{2}}|X|^{2}+11 \xi|X|^{4}\right. \\
& \left.-\frac{2}{9}\left(\frac{6}{M^{2}}-\xi|X|^{2}\right) b_{m} b^{m}\right]\left(R+\frac{2}{3} b_{m} b^{m}\right) \\
& +\left(\frac{6}{M^{2}}-\xi|X|^{2}\right)\left(\frac{1}{72} R^{2}-2 \partial_{m} X \partial^{m} \bar{X}\right. \\
& \left.+\frac{1}{18}\left(\nabla_{m} b^{m}\right)^{2}-\frac{1}{162}\left(b_{m} b^{m}\right)^{2}\right) \\
& +\frac{i}{2}(\beta-\bar{\beta}) \nabla_{m} b^{m}-\frac{i}{3}\left(\frac{12}{M^{2}}-\xi|X|^{2}\right) b^{m} \\
& \times\left(\bar{X} \partial_{m} X-X \partial_{m} \bar{X}\right)-U(X, \bar{X}),
\end{aligned}
$$

where the scalar potential $U(X, \bar{X})$ reads

$U=-6(\alpha \bar{X}+\bar{\alpha} X)-6(\beta+\bar{\beta})|X|^{2}-\frac{48}{M^{2}}|X|^{4}+18 \xi|X|^{6}$,

and we demand $\operatorname{Re} \beta<0$ for the correct sign of the EinsteinHilbert term.

The scalar potential (4) has an anti-de-Sitter (AdS) minimum unless $\alpha$ vanishes, so we set $\alpha=0$. This uplifts the minimum at $X=0$ to a Minkowski vacuum provided that the parameters are chosen appropriately (see the next sections). Then (at $X=0$ ) the canonical normalization of the Einstein-Hilbert term fixes $\beta=-1$ (or $\operatorname{Re} \beta=-1$ in general). Next, as will be shown below, the parameter $M$ will be the mass of Starobinsky scalaron, so that it can be fixed by identifying scalaron with inflaton via CMB measurements. Hence, we are left with a single free parameter $\xi$ that will determine the shape of the scalar potential.

\section{Dual supergravity}

It is remarkable that the higher-derivative modified supergravity (1) can be transformed to the standard supergravity (in Jordan frame, without higher derivatives) as was first demonstrated by Cecotti in 1987 [38], similarly to the well known duality between a modified $f(R)$ gravity and a scalar-tensor gravity. Moreover, a duality transformation can be done in the manifestly supersymmetric way, when using superspace $[36,41]$. In this section, we first apply the duality transformation to the Lagrangian (3) in the familiar field components and then dualize the whole superfield action in Eq. (1). Of course, both approaches lead to the same physics and the Lagrangians coincide after some field redefinitions, but only the superspace approach is manifestly supersymmetric.

\subsection{Dual bosonic part in field components}

Let us introduce the notation

$\frac{M^{4} \xi}{144} \equiv \zeta \quad$ and $\quad|X| \equiv \frac{M}{2 \sqrt{6}} \sigma$,

where $\sigma$ is the radial part of the complex scalar $X$. Its angular part (let us call it $\theta$ ) does not appear in the potential because we set $\alpha=0 .{ }^{3}$ We also set $\theta=b_{m}=0$ for simplicity.

Then the action (3) takes the form

$e^{-1} \mathcal{L}=\frac{1}{2} f(R, \sigma)-\frac{1}{2}\left(1-\zeta \sigma^{2}\right)(\partial \sigma)^{2}-U$,

where we find

$$
\begin{aligned}
f(R, \sigma) & =\left(1+\frac{1}{6} \sigma^{2}-\frac{11}{24} \zeta \sigma^{4}\right) R+\frac{1}{6 M^{2}}\left(1-\zeta \sigma^{2}\right) R^{2} \\
U & =\frac{1}{2} M^{2} \sigma^{2}\left(1-\frac{1}{6} \sigma^{2}+\frac{3}{8} \zeta \sigma^{4}\right) .
\end{aligned}
$$

By following the standard procedure, we introduce the auxiliary field $\chi$ and rewrite the action as

$e^{-1} \mathcal{L}=\frac{1}{2}\left[f_{\chi}(R-\chi)+f\right]-\frac{1}{2}\left(1-\zeta \sigma^{2}\right)(\partial \sigma)^{2}-U$,

where $f_{\chi} \equiv \frac{\partial f}{\partial \chi}$, and $f \equiv f(\chi, \sigma)$ is the function (7) with $R$ replaced by $\chi$. Varying with respect to $\chi$ leads to the action (6). A transfer to Einstein frame is obtained via Weyl rescaling,

$$
\begin{aligned}
g_{m n} & \rightarrow f_{\chi}^{-1} g_{m n}, \quad e \rightarrow f_{\chi}^{-2} e, \\
e f_{\chi} R & \rightarrow e R-\frac{3}{2} e f_{\chi}^{-2}\left(\partial f_{\chi}\right)^{2} .
\end{aligned}
$$

\footnotetext{
${ }^{3}$ With $\alpha=0$ our model has the global R-symmetry under which $X$ is rotated by a phase.
} 
Therefore, the function

$$
\begin{aligned}
f_{\chi} & =A+B \chi \quad \text { with } \\
A & \equiv 1+\frac{1}{6} \sigma^{2}-\frac{11}{24} \zeta \sigma^{4} \text { and } B \equiv \frac{1}{3 M^{2}}\left(1-\zeta \sigma^{2}\right)
\end{aligned}
$$

can be identified with Starobinsky scalaron that can be brought to the canonically normalized field $\varphi$ via the identification

$f_{\chi}=\exp \left[\sqrt{\frac{2}{3}} \varphi\right]$

so that

$\chi=\frac{1}{B}\left(e^{\sqrt{\frac{2}{3} \varphi}}-A\right)$ and $f=\frac{1}{2 B}\left(e^{2 \sqrt{\frac{2}{3}} \varphi}-A^{2}\right)$,

which is essentially the change of variables from $\chi$ to $\varphi$.

After the Weyl rescaling (10) the Lagrangian (9) takes the following form in terms of the canonical scalaron $\varphi$ :

$e^{-1} \mathcal{L}=\frac{1}{2} R-\frac{1}{2}(\partial \varphi)^{2}-\frac{1}{2}\left(1-\zeta \sigma^{2}\right) e^{-\sqrt{\frac{2}{3}} \varphi}(\partial \sigma)^{2}-V$,

where the two-field scalar potential reads

$$
\begin{aligned}
V= & \frac{1}{4 B}\left(1-A e^{-\sqrt{\frac{2}{3} \varphi}}\right)^{2}+e^{-2 \sqrt{\frac{2}{3} \varphi}} U \\
= & \frac{3 M^{2}}{4\left(1-\zeta \sigma^{2}\right)}\left[1-e^{-\sqrt{\frac{2}{3}} \varphi}-\frac{1}{6} \sigma^{2}\left(1-\frac{11}{4} \zeta \sigma^{2}\right) e^{-\sqrt{\frac{2}{3}} \varphi}\right]^{2} \\
& +\frac{M^{2}}{2} e^{-2 \sqrt{\frac{2}{3}} \varphi} \sigma^{2}\left(1-\frac{1}{6} \sigma^{2}+\frac{3}{8} \zeta \sigma^{4}\right) .
\end{aligned}
$$

As is clear from the Lagrangian (14), when $\sigma^{2}>1 / \zeta$, the scalar $\sigma$ becomes a ghost. However, when approaching $\sigma^{2}=1 / \zeta$, the potential (15) becomes singular, so that it would take the infinite amount of energy to turn $\sigma$ into a ghost (assuming its starting value in the region $\sigma^{2}<1 / \zeta$ ). It is also worth noticing that the inflaton mass $M$ enters the potential as the overall factor, so that it does not affect the shape of the potential.

\subsection{Superfield dual version}

As was demonstrated in Ref. [36], the dual superfield Lagrangian of Eq. (1) is obtained by introducing the Lagrange multiplier (chiral) superfield $\mathbf{T}$ as ${ }^{4}$

$$
\begin{gathered}
\mathcal{L}=\int d^{2} \Theta 2 \mathcal{E}\left\{-\frac{1}{8}\left(\overline{\mathcal{D}}^{2}-8 \mathcal{R}\right) N(\mathbf{S}, \overline{\mathbf{S}})+\mathcal{F}(\mathbf{S})\right. \\
+6 \mathbf{T}(\mathbf{S}-\mathcal{R})\}+ \text { h.c. }
\end{gathered}
$$

Varying it with respect to $\mathbf{T}$ gives back the original Lagrangian (1) by identifying the chiral superfield $\mathbf{S}$ with $\mathcal{R}$.

When using instead the superspace identity

$$
\begin{aligned}
& \int d^{2} \Theta 2 \mathcal{E}\left(\overline{\mathcal{D}}^{2}-8 \mathcal{R}\right)(\mathbf{T}+\overline{\mathbf{T}})+\text { h.c. } \\
& =-16 \int d^{2} \Theta 2 \mathcal{E} \mathcal{R} \mathbf{T}+\text { h.c. }
\end{aligned}
$$

the Lagrangian (16) can be rewritten to

$$
\begin{aligned}
\mathcal{L}= & \int d^{2} \Theta 2 \mathcal{E}\left\{\frac{3}{8}\left(\overline{\mathcal{D}}^{2}-8 \mathcal{R}\right)\left[\mathbf{T}+\overline{\mathbf{T}}-\frac{1}{3} N(\mathbf{S}, \overline{\mathbf{S}})\right]\right. \\
& +\mathcal{F}(\mathbf{S})+6 \mathbf{T S}\}+ \text { h.c. }
\end{aligned}
$$

Given the functions $N(\mathcal{R}, \overline{\mathcal{R}})$ and $\mathcal{F}(\mathcal{R})$ according to Eq. (2), the Lagrangian (18) can be rewritten to the standard form,

$\mathcal{L}=\int d^{2} \Theta 2 \mathcal{E}\left[\frac{3}{8}\left(\overline{\mathcal{D}}^{2}-8 \mathcal{R}\right) e^{-K / 3}+W\right]+$ h.c.

where the Kähler potential $K$ and the superpotential $W$ are given by (cf. Ref. [40])

$K=-3 \log (\mathbf{T}+\overline{\mathbf{T}}-\tilde{N}), \quad \tilde{N} \equiv \frac{1}{3} N=\mathbf{S} \overline{\mathbf{S}}-\frac{3}{2} \zeta(\mathbf{S} \overline{\mathbf{S}})^{2}$,

$W=3 M \mathbf{S}\left(\mathbf{T}-\frac{1}{2}\right)$,

after rescaling $\mathbf{S} \rightarrow M \mathbf{S} / 2$ and using the parameter $\zeta \equiv$ $M^{4} \xi / 144$.

It is straightforward to derive the corresponding bosonic terms in field components. We find

$$
\begin{aligned}
e^{-1} \mathcal{L}= & \frac{1}{2} R-K_{i \bar{j}} \partial_{m} \Phi^{i} \partial^{m} \bar{\Phi}^{j} \\
& \quad-e^{K}\left(K^{i \bar{j}} D_{i} W D_{\bar{j}} \bar{W}-3|W|^{2}\right),
\end{aligned}
$$

where $\Phi^{i}=(T, S), i=1,2$, and the Kähler metric reads

$$
K_{i \bar{j}}=\left(\begin{array}{cc}
K_{T \bar{T}} & K_{T \bar{S}} \\
K_{S \bar{T}} & K_{S \bar{S}}
\end{array}\right)=\frac{3}{P^{2}}\left(\begin{array}{cc}
1 & -\tilde{N}_{\bar{S}} \\
-\tilde{N}_{S} & \tilde{N}_{S} \tilde{N}_{\bar{S}}+P \tilde{N}_{S \bar{S}}
\end{array}\right)
$$

with $P \equiv T+\bar{T}-\tilde{N}$. The inverse Kähler metric is given by

$$
K^{i \bar{j}}=\left(\begin{array}{cc}
K^{T \bar{T}} & K^{T \bar{S}} \\
K^{S \bar{T}} & K^{S \bar{S}}
\end{array}\right)=\frac{P}{3}\left(\begin{array}{cc}
P+\tilde{N}^{S \bar{S}} \tilde{N}_{S} \tilde{N}_{\bar{S}} & \tilde{N}^{S \bar{S}} \tilde{N}_{S} \\
\tilde{N}^{S \bar{S}} \tilde{N}_{\bar{S}} & \tilde{N}^{S \bar{S}}
\end{array}\right) .
$$

\footnotetext{
${ }^{4}$ We use the bold font for the chiral superfields $\mathbf{S}$ and $\mathbf{T}$, and the regular font for their leading field components.
} 
Because of the non-vanishing non-diagonal elements of the Kähler metric, the kinetic part of the Lagrangian mixes the derivatives of $S$ and $T$,

$$
\begin{aligned}
e^{-1} \mathcal{L}_{\text {kin }}= & -\frac{3}{P^{2}}\left[\partial T \partial \bar{T}-\tilde{N}_{S} \partial S \partial \bar{T}-\tilde{N}_{\bar{S}} \partial T \partial \bar{S}\right. \\
& \left.+\left(\tilde{N}_{S} \tilde{N}_{\bar{S}}+P \tilde{N}_{S \bar{S}}\right) \partial S \partial \bar{S}\right] .
\end{aligned}
$$

In order to bring the Lagrangian to the form (14), where the contributions of $b_{m}{ }^{5}$ and the angular part of $X$ are ignored, we set $\operatorname{Im} T=0, S=|S|$, and denote $|S|=\sigma / \sqrt{6}$. The kinetic mixing between $\partial S$ and $\partial T$ can be eliminated by using $P=T+\bar{T}-\tilde{N}$ as the independent (real) scalar instead of $\operatorname{Re} T$. We get the canonical normalization of its kinetic term in the parametrization $P=\exp \left[\sqrt{\frac{2}{3}} \varphi\right]$ as follows:

$e^{-1} \mathcal{L}_{\text {kin }}=-\frac{1}{2}(\partial \varphi)^{2}-\frac{1}{2}\left(1-\zeta \sigma^{2}\right) e^{-\sqrt{\frac{2}{3}} \varphi}(\partial \sigma)^{2}$,

that exactly matches the kinetic part of Eq. (14).

It is also straightforward (albeit tedious) to check that the scalar potential of Eq. (22) also coincides with that of Eq. (15) after using the field redefinitionsqa diagonalizing the scalar kinetic matrix above.

\section{Critical points}

To study vacuum equations in our basic model, we denote $e^{-\sqrt{\frac{2}{3} \varphi}} \equiv x$ and rewrite the scalar potential (15) as

$$
\begin{aligned}
& V=\frac{1}{4 B}(1-A x)^{2}+x^{2} U, \\
& \text { where }\left\{\begin{array}{l}
A=1+\frac{1}{6} \sigma^{2}-\frac{11}{24} \zeta \sigma^{4}, \\
B=\frac{1}{3 M^{2}}\left(1-\zeta \sigma^{2}\right), \\
U=\frac{M^{2}}{2} \sigma^{2}\left(1-\frac{1}{6} \sigma^{2}+\frac{3}{8} \zeta \sigma^{4}\right) .
\end{array}\right.
\end{aligned}
$$

The equations for critical points read

$$
\begin{aligned}
\partial_{x} V & =\frac{A}{2 B}(A x-1)+2 x U=0, \\
\partial_{\sigma} V & =\frac{2 x A^{\prime} B+(1-A x) B^{\prime}}{4 B^{2}}(A x-1)+x^{2} U^{\prime}=0,
\end{aligned}
$$

where the primes denote the derivatives with respect to $\sigma$. A simple solution to these equations is

$$
A x=1, \quad U=U^{\prime}=0 .
$$

\footnotetext{
5 The vector field $b_{m}$ in higher-derivative supergravity contributes a physical scalar $\nabla_{m} b^{m}$, as was shown in Ref. [36]. In the dual mattercoupled supergravity, this scalar can be associated with the axionic field $\operatorname{Im} T$.
}

It gives rise to the vanishing potential (27) for $\sigma_{0}=\varphi_{0}=0$. There is another solution by taking $U^{\prime}=0$ and obtaining

$\sigma^{2}=\frac{2}{27 \zeta}(2 \pm \sqrt{4-162 \zeta})$.

On the other hand, the condition $U=0$ is solved by

$\sigma^{2}=\frac{2}{9 \zeta}(1 \pm \sqrt{1-54 \zeta})$.

Equating Eqs. (31) and (32) leads to an equation on the parameter $\zeta$ with a solution $\zeta=1 / 54 \approx 0.019$ provided that the "plus" branch is chosen in Eq. (31). It means, when $\zeta=1 / 54$, we have three Minkowski minima: $\sigma_{0}$ and $\pm\left|\sigma_{1}\right|$ where $\sigma_{1}^{2}$ is given by Eqs. (31) or (32).

When $\zeta \neq 1 / 54$, the two minima at $\pm \sigma_{1}$ are not given by Eqs. (31) and (32), being more general solutions to the vacuum equations (28) and (29). In particular, when $0<\zeta<$ $1 / 54$, the minima at $\pm \sigma_{1}$ are AdS, while for $1 / 54<\zeta<$ 0.027 the minima are uplifted to metastable de-Sitter $(\mathrm{dS})$. When $\zeta \approx 0.027$, there are two inflection points, whereas for $\zeta>0.027$ all the critical points, except of $\sigma=0$, disappear. The scalar potential $V / M^{2}$ is shown in Fig. 1a (at $\zeta=1 / 54$ ) and Fig. 1b (at $\zeta=0.027$ ).

Let us comment on the scalar masses for the model (20) and (21). Expanding around the Minkowski vacuum at $\varphi=$ $\sigma=0$, we find $M_{\varphi}=M_{\sigma}=M$, where $M_{\varphi}$ and $M_{\sigma}$ are the masses of $\varphi$ and $\sigma$, respectively. As for the axion $\operatorname{Im} T$, after its proper normalization we find that it also has the mass $M$. However, the last scalar $\theta$ has vanishing mass around the minimum, so it must be generated by additional means. This, together with the more detailed analysis of the dynamics of $\operatorname{Im} T$ and $\theta$ deserves a separate investigation that we leave to future works. Here we will focus on the two scalars $\varphi$ and $\sigma$.

\section{Two-field inflationary dynamics}

Having derived the Lagrangian with the two-field scalar potential from the modified supergravity, in this section we investigate its suitability for describing cosmological inflation in agreement with $\mathrm{CMB}$ observations.

\subsection{Field equations}

The Lagrangian in Eqs. (14) and (15) takes the form of a nonlinear sigma-model (NLSM) minimally coupled to gravity,

$e^{-1} \mathcal{L}=\frac{1}{2} R-\frac{1}{2} G_{A B} \partial \phi^{A} \partial \phi^{B}-V$,

where $\phi^{A}=\{\varphi, \sigma\}, A=1,2$, and the NLSM metric is given by

$G_{A B}=\left(\begin{array}{lc}1 & 0 \\ 0 & \left(1-\zeta \sigma^{2}\right) e^{-\sqrt{\frac{2}{3}} \varphi}\end{array}\right)$. 


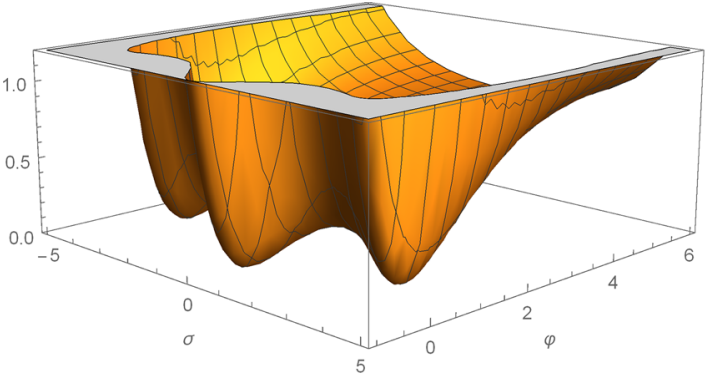

(a)

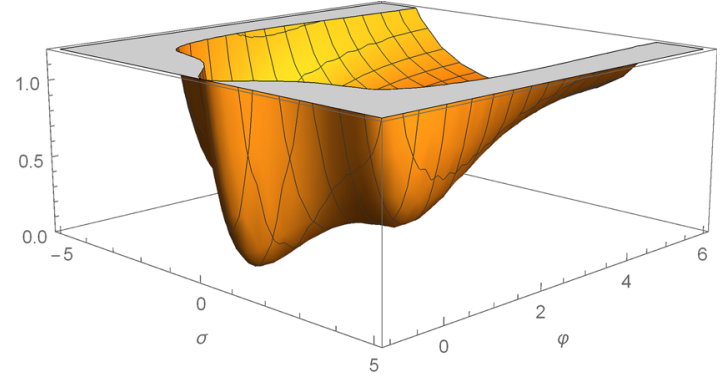

(b)

Fig. 1 The scalar potential $V / M^{2}$ of Eq. (27). The plot $\mathbf{a} \zeta=1 / 54 \approx 0.019$ with three Minkowski minima. The plot $\mathbf{b} \zeta=0.027$ with a single Minkowski minimum at $\sigma=0$ and two inflection points

Varying the Lagrangian (33) with respect to the scalar fields yields equations of motion in the form

$\square \phi^{C}+\Gamma_{A B}^{C} \partial \phi^{A} \partial \phi^{B}=G^{A C} \partial_{A} V$

where $\square \equiv \nabla_{m} \nabla^{m}$ is the spacetime Laplace-Beltrami operator, and $\Gamma_{A B}^{C}$ are the Christoffel symbols of the NLSM target space. The non-vanishing Christoffel symbols are

$\Gamma_{\sigma \varphi}^{\sigma}=-\frac{1}{\sqrt{6}}, \quad \Gamma_{\sigma \sigma}^{\varphi}=\frac{1}{\sqrt{6}}\left(1-\zeta \sigma^{2}\right) e^{-\sqrt{\frac{2}{3} \varphi}}$,

$\Gamma_{\sigma \sigma}^{\sigma}=-\frac{\zeta \sigma}{1-\zeta \sigma^{2}}$.

After using these results, and the Friedmann-LemaitreRobertson-Walker (FLRW) spacetime metric $g_{m n}=$ $\operatorname{diag}\left(-1, a^{2}, a^{2}, a^{2}\right)$ with the time-dependent scale factor $a(t)$, the equations of motion take the form

$\ddot{\varphi}+3 H \dot{\varphi}+\frac{1}{\sqrt{6}}\left(1-\zeta \sigma^{2}\right) e^{-\sqrt{\frac{2}{3} \varphi}} \dot{\sigma}^{2}+\partial_{\varphi} V=0$,

$\ddot{\sigma}+3 H \dot{\sigma}-\frac{\zeta \sigma \dot{\sigma}^{2}}{1-\zeta \sigma^{2}}-\sqrt{\frac{2}{3}} \dot{\varphi} \dot{\sigma}+\frac{e^{\sqrt{\frac{2}{3} \varphi}}}{1-\zeta \sigma^{2}} \partial_{\sigma} V=0$,

where the dots stand for the time derivatives.

The Friedmann equations for the system (33) read

$$
\begin{aligned}
3 H^{2} & =\frac{1}{2} \dot{\varphi}^{2}+\frac{1}{2}\left(1-\zeta \sigma^{2}\right) e^{-\sqrt{\frac{2}{3} \varphi}} \dot{\sigma}^{2}+V, \\
\dot{H} & =-\frac{1}{2} \dot{\varphi}^{2}-\frac{1}{2}\left(1-\zeta \sigma^{2}\right) e^{-\sqrt{\frac{2}{3} \varphi}} \dot{\sigma}^{2},
\end{aligned}
$$

where the Hubble function has been introduced, $H \equiv \dot{a} / a$.

For numerical computations it is useful to rescale time as $\tilde{t} \equiv M t$ (when using $\tilde{t}$, the dots will denote the derivatives with respect to $\tilde{t}$ ) with the rescaled Hubble function $\tilde{H}=$ $H / M$.

\subsection{Inflationary parameters}

In this Subsection we employ the covariant formalism that is well known in the literature, see e.g., Refs. [42-44] and the references therein, with the slow-roll parameter

$\epsilon \equiv-\frac{\dot{H}}{H^{2}}=-\frac{\dot{\tilde{H}}}{\tilde{H}^{2}}$.

In a two-field analysis, it is useful to define the field-space velocity and acceleration (turn rate) unit vectors as

$\Sigma^{A} \equiv \frac{\dot{\phi}^{A}}{|\dot{\phi}|}, \quad \Omega^{A} \equiv \frac{\omega^{A}}{|\omega|}$,

respectively, where the absolute value of a field-space vector $a^{A}$ is defined by $|a| \equiv \sqrt{G_{A B} a^{A} a^{B}}$, and the acceleration vector $\omega^{A}$ is defined by

$$
\begin{aligned}
\omega^{A} \equiv & \dot{\Sigma}^{A}+\Gamma_{B C}^{A} \Sigma^{B} \dot{\phi}^{C} \\
& \left\{\begin{array}{l}
\omega^{\varphi}=\dot{\Sigma}^{\varphi}+\frac{1}{\sqrt{6}}\left(1-\zeta \sigma^{2}\right) e^{-\sqrt{\frac{2}{3}} \varphi} \Sigma^{\sigma} \dot{\sigma}, \\
\omega^{\sigma}=\dot{\Sigma}^{\sigma}-\frac{1}{\sqrt{6}}\left(\Sigma^{\varphi} \dot{\sigma}+\Sigma^{\sigma} \dot{\varphi}\right)-\frac{\zeta \sigma}{1-\zeta \sigma^{2}} \Sigma^{\sigma} \dot{\sigma} .
\end{array}\right.
\end{aligned}
$$

Another useful quantity is the effective mass matrix,

$\mathcal{M}_{B}^{A} \equiv G^{A C} \nabla_{B} \partial_{C} V-R_{C D B}^{A} \dot{\phi}^{C} \dot{\phi}^{D}$,

where $R_{C D B}^{A}$ is the Riemann tensor of the NLSM scalar manifold, with the non-vanishing components

$R_{\sigma \sigma \varphi}^{\varphi}=\frac{1}{6}\left(1-\zeta \sigma^{2}\right) e^{-\sqrt{\frac{2}{3} \varphi}}, \quad R_{\varphi \varphi \sigma}^{\sigma}=\frac{1}{6}$.

With the above definitions we can introduce the adiabatic and isocurvature parameters

$\eta_{\Sigma \Sigma} \equiv \frac{\mathcal{M}_{B}^{A} \Sigma_{A} \Sigma^{B}}{V}, \quad \eta_{\Omega \Omega} \equiv \frac{\mathcal{M}_{B}^{A} \Omega_{A} \Omega^{B}}{V}$,

respectively, where $\eta_{\Sigma \Sigma}$ plays the role of the second slow-roll parameter, while $\eta_{\Omega \Omega}$ is proportional to the effective isocurvature mass. 


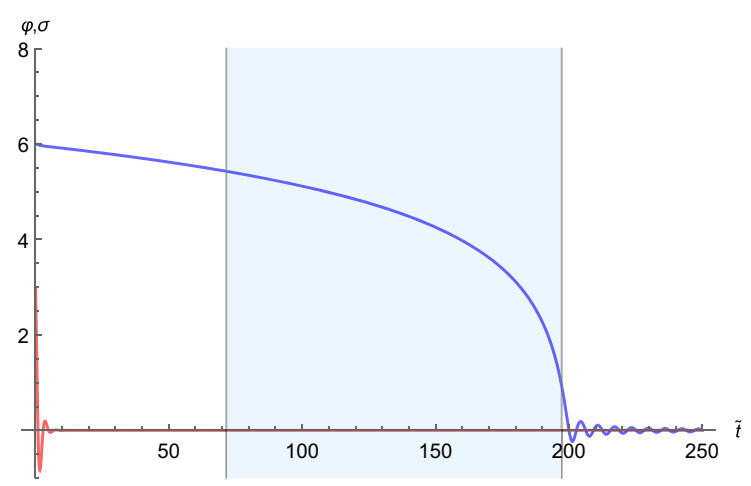

(a) The solutions $\varphi(\tilde{t})$ (blue) and $\sigma(\tilde{t})$ (red).

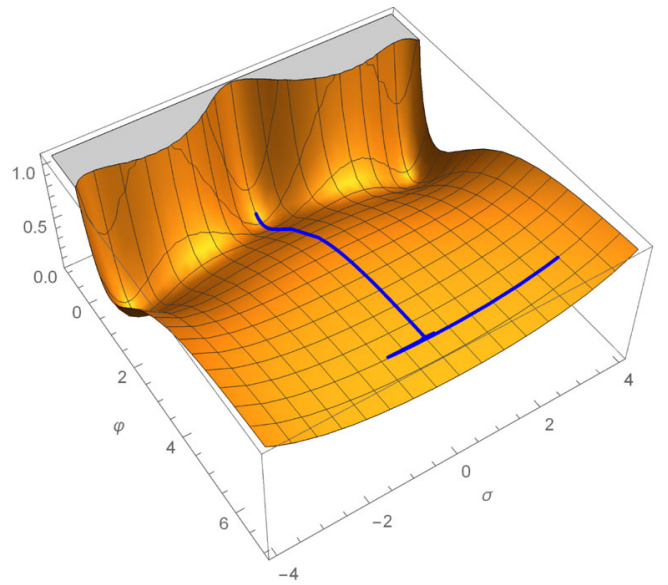

(b) The field-space trajectory of the solutions.

Fig. 2 The solutions (I) to the field equations (37) and (38) with the initial conditions $\varphi(0)=6, \sigma(0)=3$ and the vanishing initial velocities. The blue shaded region represents the time period of the last 60 e-foldings

The transfer functions are defined as follows:

$T_{S S}\left(t_{1}, t_{2}\right) \equiv \exp \left[\int_{t_{1}}^{t_{2}} d t^{\prime} \beta\left(t^{\prime}\right) H\left(t^{\prime}\right)\right]$,
$T_{R S}\left(t_{1}, t_{2}\right) \equiv 2 \int_{t_{1}}^{t_{2}} d t^{\prime}\left|\omega\left(t^{\prime}\right)\right| T_{S S}\left(t_{1}, t_{2}\right)$,

where

$\beta(t) \equiv-2 \epsilon+\eta_{\Sigma \Sigma}-\eta_{\Omega \Omega}-\frac{4|\omega|^{2}}{3 H^{2}}$

The transfer functions describe the evolution of perturbations on superhorizon scales, i.e. from the moment of horizon exit $t_{1}$ (of the $k$-mode of interest) until some later time $t_{2}$.

The inflationary observables (CMB tilts) can be computed as (by assuming that isocurvature modes are suppressed)

$n_{s}=1-6 \epsilon+2 \eta_{\Sigma \Sigma}$ and $r=\frac{16 \epsilon}{1+T_{R S}^{2}}$.

As $T_{R S}$ is real, the maximum value of the tensor-to-scalar ratio is $r_{\max }=16 \epsilon$, while it can be computed without the transfer functions. In Appendix B we estimate both $T_{\mathrm{RS}}$ and $T_{\mathrm{SS}}$ and find them negligible. Therefore, isocurvature effects can be ignored at CMB scales indeed.

According to the latest PLANCK data [21], the observed values of $n_{s}$ and $r$ are

$n_{s}=0.9649 \pm 0.0042(1 \sigma \mathrm{CL})$ and $r<0.064(2 \sigma \mathrm{CL})$.

\subsection{Inflationary solutions}

Let us first consider the case of $\zeta=1 / 54 \approx 0.019$ with three Minkowski minima in Fig. 1a.
We numerically solve the field equations (37), (38) and (39) with the initial conditions $\varphi(0)=6, \sigma(0)=3$, and the vanishing initial velocities, so let us call it the solution (I). The scalar field solutions are plotted in Fig. 2a, and their trajectories in the scalar potential are plotted in Fig. 2b. It can be seen that $\sigma$ quickly drops to its minimum $\sigma=0$, so that the trajectory becomes similar to that in the single-field Starobinsky inflation. In fact, this is a generic feature when the initial velocities are zero (or almost zero), $\varphi(0) \gtrsim 6$ and $|\sigma(0)| \lesssim \sigma_{\max }$, where $\sigma_{\max }=1 / \sqrt{\zeta}$ is the upper bound on $\sigma$ where the potential is infinite. When $\zeta=1 / 54$ we find $\sigma_{\max } \approx 7.35$.

The solution (I) leads to the spectral tilt and the tensorto-scalar ratio as $n_{s} \approx 0.9624$ and $r_{\max } \approx 0.004,{ }^{6}$ which are consistent with the observed values and the theoretical (Starobinsky) predictions of chaotic single-field inflation.

As the initial value $\sigma(0)$ approaches $\sigma_{\max }$ and/or as the initial velocities become non-negligible, the trajectory starts to curve. Also a smaller value of $\varphi(0)$ makes it easier to curve the trajectory.

As regards $\mathrm{PBH}$ production after inflation, let us consider the field-space trajectory going through the saddle point of the potential, that is a maximum in the $\sigma$-direction and a (local) minimum in the $\varphi$-direction. Then the saddle point divides inflation into two stages. We found a set of initial conditions that leads to such trajectory with

$\varphi(0)=5, \quad \dot{\sigma}(0)=79.784527415607, \sigma(0)=\dot{\varphi}(0)=0$.

\footnotetext{
${ }^{6}$ We evaluate $n_{s}$ and $r$ at the CMB pivot scale $k=0.05 \mathrm{Mpc}^{-1}$ identified with the scale exiting the horizon around 54 e-folds before the end of inflation, and assume the standard reheating temperature of the order $10^{9} \mathrm{GeV}$ [45] that is also expected in the modified supergravity setup [46].
} 


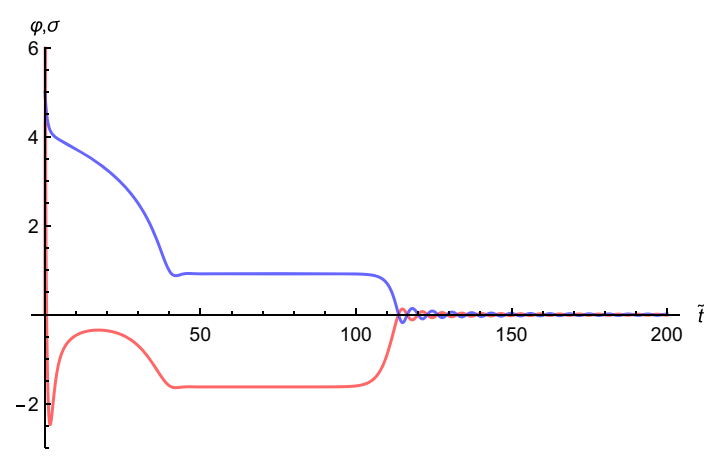

(a) The solutions $\varphi(\tilde{t})$ (blue) and $\sigma(\tilde{t})$ (red).

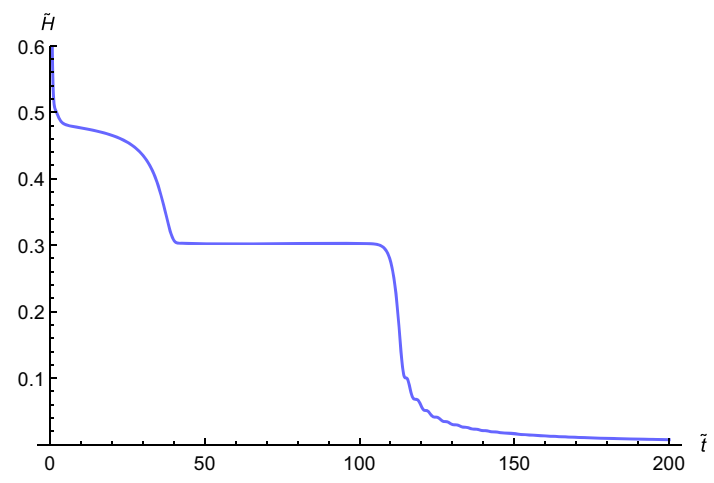

(c) The Hubble function $\tilde{H}(\tilde{t})$.

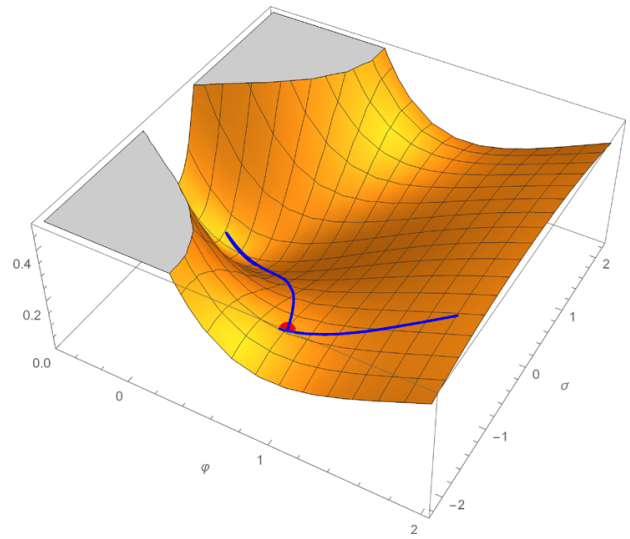

(b) The field-space trajectory of the solutions.

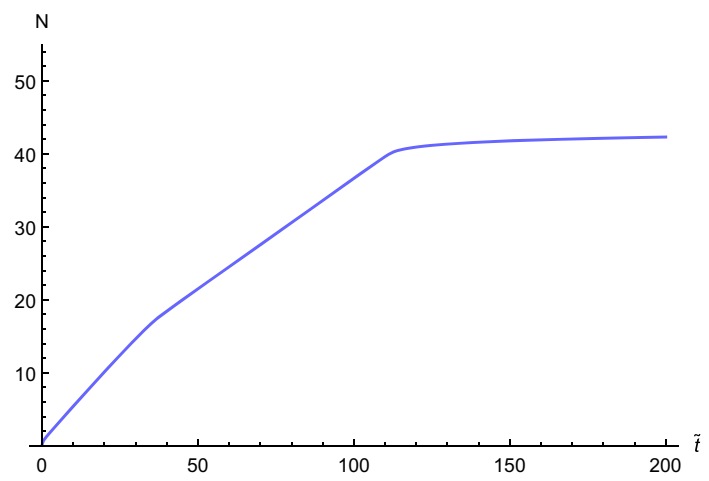

(d) The e-foldings number $N(\tilde{t})$.

Fig. 3 The solutions (II) to the field equations (37) and (38) with the initial conditions (52). The red spot in (3b) represents one of the two saddle points of the potential

Let us call the corresponding solution as the solution (II). We include its plots in Fig. 3a, b. The time-dependence of the Hubble function and the e-foldings number defined by $\dot{N}=H$ are shown in Fig. 3c, d. The total number of efoldings is around 40 , though it can be larger for larger values of $\varphi(0)$ with more fine-tuning of the initial velocities.

Thus, in order to achieve the two-stage inflation, where the field-space trajectory passes through the saddle point, we have to fine-tune the initial conditions as in Eq. (52), though the last choice is not unique. The reason is, when $\varphi$ is large, the potential takes the shape of a valley with the minima at $\sigma=0$, so a generic behavior of $\sigma$ is to quickly relax at $\sigma=0$, and let $\varphi$ drive the entire inflationary period. The same remains true if we change the shape of the potential as in Fig. $1 \mathrm{~b}$ by changing $\zeta$ (the only difference is the saddle point to be replaced by an inflection point).

\section{Generalized attractor-type models}

Having learned the lessons in the previous Sections, we conclude that our basic ansatz in Eq. (2) for functions $N(\mathcal{R}, \overline{\mathcal{R}})$ and $\mathcal{F}(\mathcal{R})$ is too restrictive because it requires extreme fine tuning of the initial conditions for PBHs production. Therefore, we generalize our ansatz by adding the next-order corrections as

$$
\begin{aligned}
& N=\frac{12}{M^{2}}|\mathcal{R}|^{2}-\frac{72}{M^{4}} \zeta|\mathcal{R}|^{4}-\frac{768}{M^{6}} \gamma|\mathcal{R}|^{6}, \\
& \mathcal{F}=-3 \mathcal{R}+\frac{3 \sqrt{6}}{M} \delta \mathcal{R}^{2},
\end{aligned}
$$

where we have introduced two new parameters $\gamma$ and $\delta$ with their normalization chosen for later convenience. We keep $\alpha=0, \beta=-3$ and $\zeta \equiv M^{4} \xi / 144$, ignore $b_{m}$ and the angular mode of $\mathcal{R} \mid=X$, and set $X=M \sigma / \sqrt{24}$, as in the previous Sections. In the framework of the dual matter-coupled supergravity (19), the $\gamma$-term resides in the Kähler potential that can be affected by quantum corrections, whereas the $\delta$-term resides in the superpotential that does not receive (perturbative) quantum corrections. 
After repeating the procedure outlined in Sect. 3.1, we obtain the Einstein frame Lagrangian as follows:

$$
\begin{aligned}
e^{-1} \mathcal{L}= & \frac{1}{2} R-\frac{1}{2}(\partial \varphi)^{2}-\frac{3 M^{2}}{2} B e^{-\sqrt{\frac{2}{3}} \varphi}(\partial \sigma)^{2} \\
& -\frac{1}{4 B}\left(1-A e^{-\sqrt{\frac{2}{3}} \varphi}\right)^{2}-e^{-2 \sqrt{\frac{2}{3}} \varphi} U,
\end{aligned}
$$

where the functions $A, B, U$ are given by

$$
\begin{aligned}
A & =1-\delta \sigma+\frac{1}{6} \sigma^{2}-\frac{11}{24} \zeta \sigma^{4}-\frac{29}{54} \gamma \sigma^{6}, \\
B & =\frac{1}{3 M^{2}}\left(1-\zeta \sigma^{2}-\gamma \sigma^{4}\right), \\
U & =\frac{M^{2}}{2} \sigma^{2}\left(1+\frac{1}{2} \delta \sigma-\frac{1}{6} \sigma^{2}+\frac{3}{8} \zeta \sigma^{4}+\frac{25}{54} \gamma \sigma^{6}\right) .
\end{aligned}
$$

When $\gamma=\delta=0$, all that reduces to Eqs. (14) and (15), as it should. Similarly to the basic model, there is the infinite wall in the scalar potential, which prevents $\sigma$ from obtaining values leading to the wrong sign of its kinetic term.

The relevant field equations of the generalized model are

$$
0=\ddot{\varphi}+3 H \dot{\varphi}+\frac{1}{\sqrt{6}}\left(1-\zeta \sigma^{2}-\gamma \sigma^{4}\right) e^{-\sqrt{\frac{2}{3}} \varphi} \dot{\sigma}^{2}+\partial_{\varphi} V
$$

$$
0=\ddot{\sigma}+3 H \dot{\sigma}-\frac{\zeta \sigma+2 \gamma \sigma^{3}}{1-\zeta \sigma^{2}-\gamma \sigma^{4}} \dot{\sigma}^{2}-\sqrt{\frac{2}{3}} \dot{\varphi} \dot{\sigma}
$$

$$
+\frac{e^{\sqrt{\frac{2}{3}} \varphi}}{1-\zeta \sigma^{2}-\gamma \sigma^{4}} \partial_{\sigma} V
$$

$0=\frac{1}{2} \dot{\varphi}^{2}+\frac{1}{2}\left(1-\zeta \sigma^{2}-\gamma \sigma^{4}\right) e^{-\sqrt{\frac{2}{3} \varphi} \dot{\sigma}^{2}+\dot{H},}$

$0=V-3 H^{2}-\dot{H}$.

The generalized model defined by Eqs. (53) and (54) appears to be rather complicated for a detailed numerical analysis, so we study only two special cases, the one with $\delta=0$ (dubbed the $\gamma$-extension) and the one with $\gamma=0$ (dubbed the $\delta$-extension), in what follows.

\subsection{The $\gamma$-extension}

As a representative of the $\gamma$-extension $(\delta=0)$, we choose the parameters $\gamma=1$ and $\zeta=-1.7774$, see Fig. 4. This choice is interesting because the scalar potential (for $\varphi \gg$ 1) has two valleys where $\sigma \neq 0$, and a single Minkowski minimum at $\sigma=\varphi=0$. The first slow-roll (SR) inflation is possible along either of the valleys. The valleys merge into the Minkowski minimum by passing through inflection points (or near-inflection points) followed by the second, ultra-slowroll (USR), inflationary stage. ${ }^{7}$

After numerically solving the equations of motion (57)(60) we plot the solutions in Fig. 5. The total number of (observable) e-foldings is set to $\Delta N=60$, and the end of the first stage of inflation is defined by the time when $\eta_{\Sigma \Sigma}$ first crosses unity (see Fig. 5e). We could also define the end of the first stage from the local maximum of $\epsilon$, which nearly coincides with the former definition. As may be expected from the USR period $\epsilon_{\mathrm{USR}} \ll \epsilon_{\mathrm{SR}}$ and can be seen in Fig. 5e, it leads to an enhancement in the scalar power spectrum indeed. Inflation ends when $\epsilon=1$, as usual. With the chosen parameters, the first stage lasts $\Delta N_{1}=50$ e-foldings, whereas the second stage lasts for $\Delta N_{2}=10$ : in the subsequent figures the first stage of inflation is represented by the blue shaded region, whereas the second stage is marked by the green shaded region, whenever is relevant. The length of the second stage is controlled by the parameter $\zeta$ for a given $\gamma$.

By using Eq. (50), we find the observables at the CMB scale as

$n_{s} \approx 0.9545$ and $r_{\max } \approx 0.006$

The parameter space The parameter choice leading to a scalar potential with the suitable properties (as described above) is not unique, and for any $\gamma$ greater than $\sim 0.004$ there is a value of $\zeta$ that leads to a similar shape of the potential (with two inflection points, unique Minkowski minimum, etc.). For a given $\gamma$, one can solve the system of equations

$\partial_{\varphi} V=\partial_{\sigma} V=\mathbf{H}=0$,

where $\mathbf{H}$ is the Hessian determinant of the potential, in order to obtain the value of $\zeta$ leading to the desired inflection points. Then, by fine-tuning $\zeta$ around that value, one can change a duration of the USR stage $\Delta N_{2}$.

In order to see how $\gamma$ changes the shape of the scalar potential, let us evaluate the ratio $V_{\text {inflec. }} / V_{\infty}$ as a function of $\gamma$, where $V_{\text {inflec. }}$ is the value of the potential at an inflection point, and $V_{\infty}$ is the asymptotic value of the potential when $\varphi \rightarrow \infty$ and $\sigma$ is at its local minimum, which corresponds to the SR stage. This ratio represents the depth of the inflection points relative to the SR valleys, and it does not significantly change the curvature of the inflationary path in the $\varphi-\sigma$ plane. The plot of $V_{\text {inflec. }} / V_{\infty}$ versus $\gamma$, as well as the trajectory in the $\gamma-\zeta$ plane, which solves Eq. (62), are shown in Fig. 6. After taking all that into account, we conclude the control over the overall shape of the potential is limited due to the attractor-type behavior of $V_{\text {inflec. }} / V_{\infty}$ at large $\gamma$.

When $\gamma=1$, a solution to Eq. (62) gives $\zeta \approx-1.774$ that, in turn, leads to $\Delta N_{2} \approx 6.3$. However, in our example we

\footnotetext{
7 Actually, despite the name, during an ultra-slow-roll regime, the scalar field(s) roll down the potential faster than during a slow-roll regime (see e.g. Ref. [47]).
} 

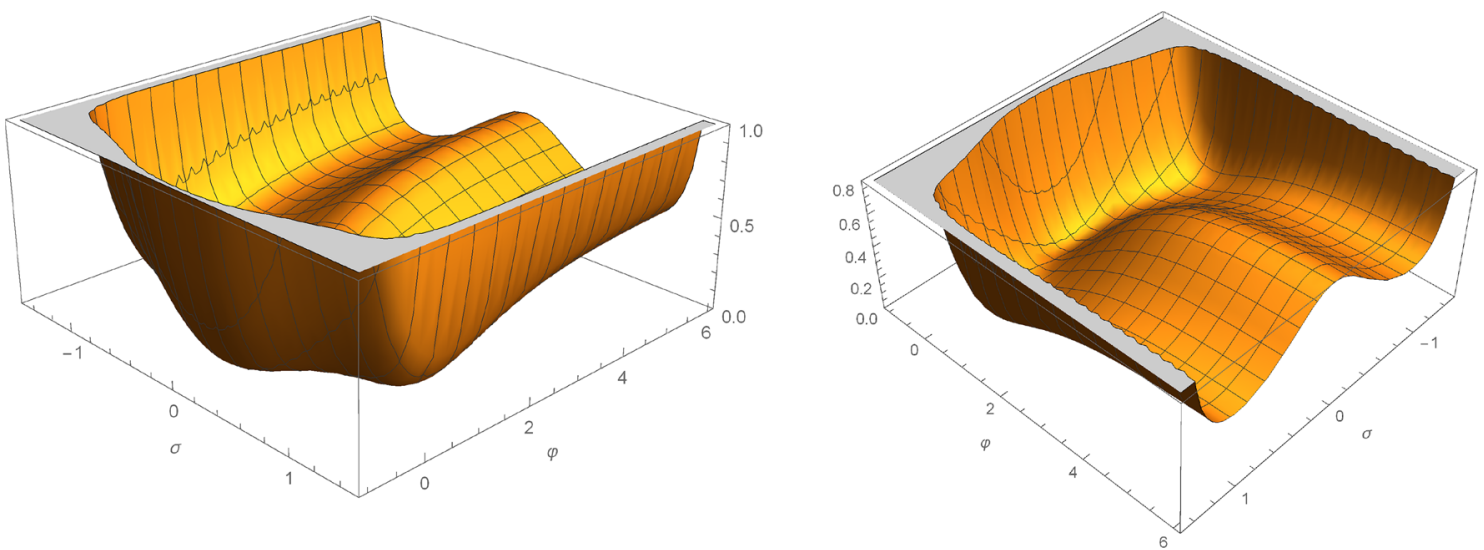

Fig. 4 The scalar potential $V / M^{2}$ in the Lagrangian (55) for $\delta=0, \gamma=1, \zeta=-1.7774$

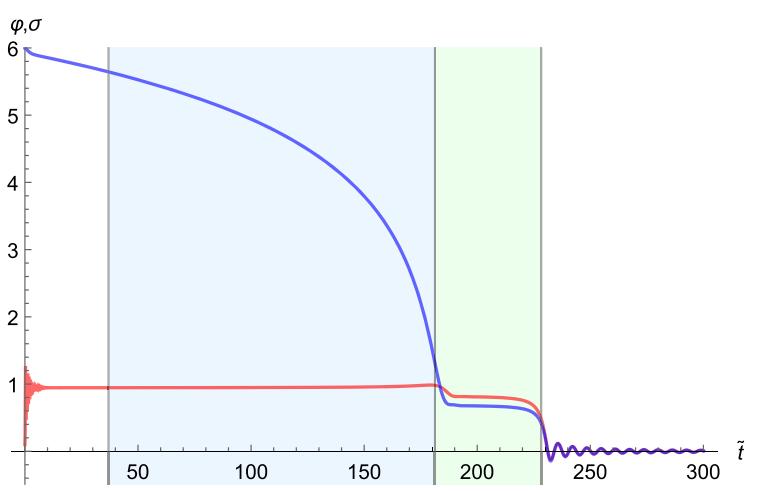

(a)

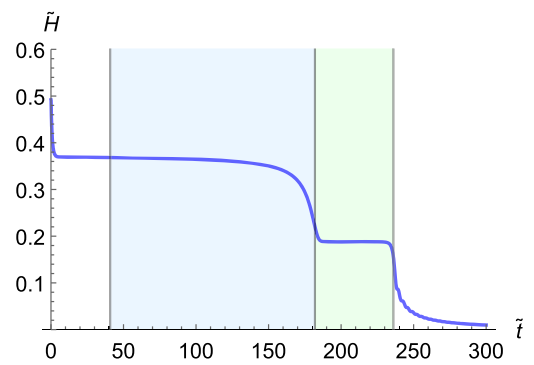

(c)

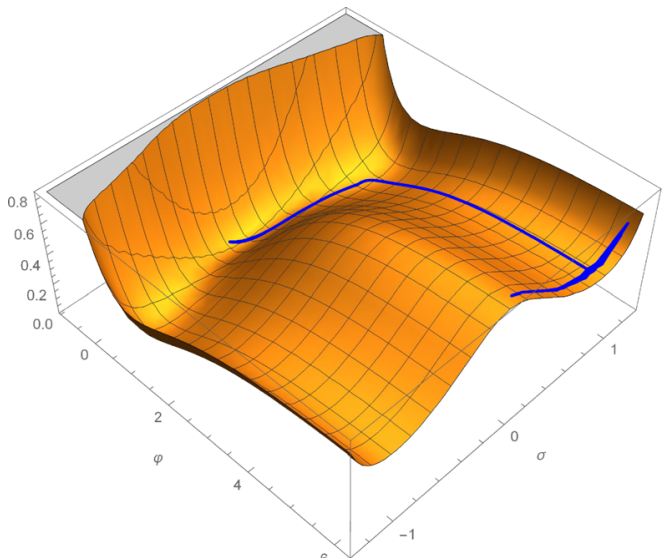

(b)

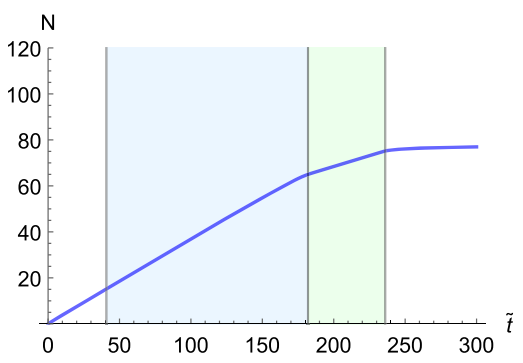

(d)

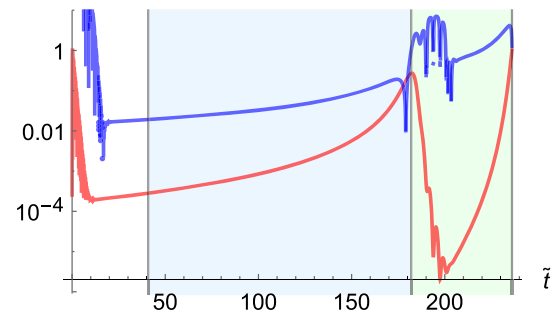

(e)
Fig. 5 a The solution to the field equations (57) and (58) with the initial conditions $\varphi(0)=6, \sigma(0)=0.1$, the vanishing initial velocities, and the choice of the parameters as $\delta=0, \gamma=1, \zeta=-1.7774$. The blue shaded region represents the first stage of inflation, and the green

slightly departed from that value of $\zeta$ and set $\zeta=-1.7774$ in order to obtain $\Delta N_{2}=10$. Our strategy is to compute power spectra for different choices of $\gamma$ while keeping $\Delta N_{2}=10$. The latter condition fixes the value of $\zeta$. Next, we examine the impact of a variation of $\Delta N_{2}$.

The power spectrum at fixed $\Delta N_{2}$ We numerically compute the power spectrum of curvature perturbations by using shaded region represents the second stage. $\mathbf{b}$ The trajectory of the solution. $\mathbf{c}$ The corresponding Hubble function. $\mathbf{d}$ The e-foldings number. $\mathbf{e}$ The slow-roll parameters $\epsilon$ (red) and $\eta_{\Sigma \Sigma}$ (blue)

the transport method introduced in Refs. [48,49] with the Mathematica package described in Ref. [50]. We compute the spectrum around the pivot scale $k_{*}$ that leaves the horizon at the end of the first stage, i.e. $\Delta N_{2}$ e-folds before the end of inflation (let us call this scale $k_{\Delta N_{2}}$ ). The inflaton mass is adjusted in each case around $0.6 \times 10^{-5} M_{\mathrm{Pl}}$ by requiring $P_{\zeta} \approx 2 \times 10^{-9}$ at the CMB scale. 


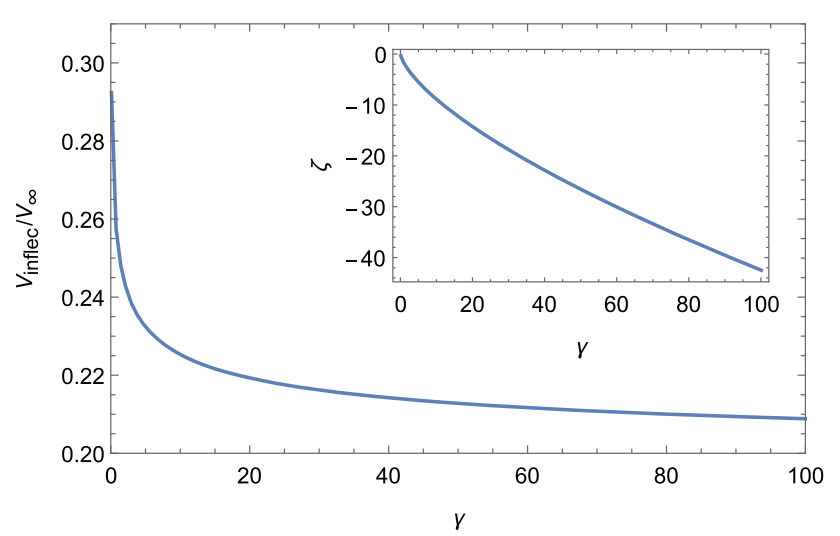

Fig. 6 The ratio of the scalar potential at the inflection point to its asymptotic value when $\varphi \rightarrow \infty$, as a function of $\gamma$. The embedded plot represents the solution to Eq. (62) in terms of $\zeta(\gamma)$

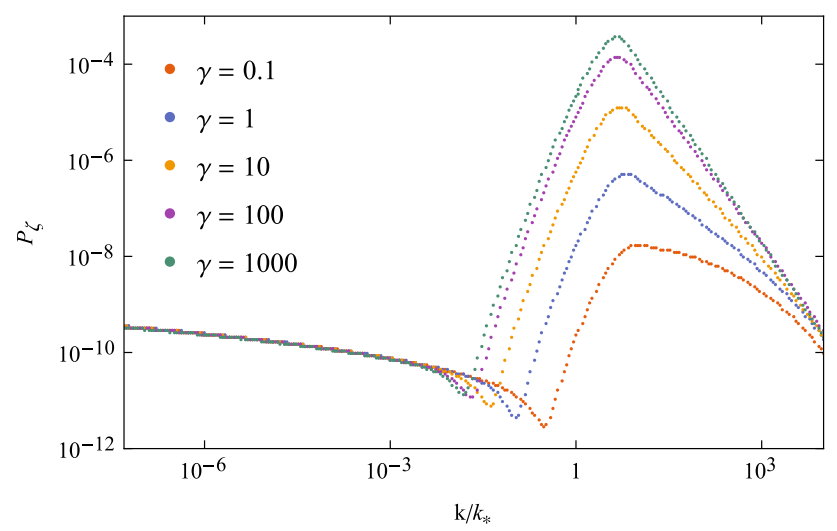

Fig. 7 The power spectrum $P_{\zeta}$ around the pivot scale $k_{*}=k_{\Delta N_{2}}$ at $\Delta N_{2}=10$ for several values of $\gamma$

Table 1 The parameters used in a computation of the power spectrum in Fig. 7 with $\Delta N_{2}=10$

\begin{tabular}{llllll}
\hline$\gamma$ & 0.1 & 1 & 10 & 100 & 1000 \\
$\zeta$ & -0.31165 & -1.7774 & -8.91495 & -42.7976 & -201.722 \\
\hline
\end{tabular}

The power spectrum for various values of $\gamma$ is shown in Fig. 7. The parameters considered are collected in Table 1, where $\zeta$ is tuned to satisfy $\Delta N_{2}=10$. A change of $n_{s}$ and $r_{\max }$ (still given by Eq. (61)) is negligible for those parameters.

As is often adopted in the literature, the desired enhancement of primordial curvature perturbations should exceed the CMB scales by the factor of $10^{7}$, in order to efficiently produce PBHs, although the authors of Ref. [15] argued by using peak theory that, given a broad peak, the required enhancement in the power spectrum drops by one order of the magnitude to $\sim 10^{6}$. Our numerical estimates with $\Delta N_{2}=10$ (see Fig. 7) show that the required enhancement of the power spectrum is not achieved. However, the enhancement grows as we increase $\Delta N_{2}$ (see below).
Changing $\Delta N_{2}$ Let us examine how the power spectrum changes with the duration of the USR regime $\Delta N_{2}$. To demonstrate that dependence, we consider the power spectrum at $\gamma=0.1$ and $\gamma=1$ with various values of duration of the USR stage, $\Delta N_{2}=10,17,20,23$ for each $\gamma$. The results are collected in Fig. 8. The case of $\gamma \lesssim 0.1$ can be excluded because the power spectrum peak is too small (technically, a larger enhancement is still possible but requires a very long USR stage that pushes the spectral index well outside of the $3 \sigma$ (lower) limit of $n_{s} \approx 0.946$ ( $c f$. Refs. [14,51]). In the case of $\gamma=1$, the required enhancement is possible provided that $\Delta N_{2} \gtrsim 20$, and, therefore, the values of $\gamma \gtrsim 1$ are favored for efficient production of PBHs.

In Table 2 we collect the approximate values of $n_{s}$ and $r_{\text {max. }}$ (at the CMB scales) for the values of $\Delta N_{2}=$ $10,17,20,23$, universally across the considered values of $\gamma=0.1,1,10,100,1000$. The tensor-to-scalar ratio $r$ is well within the observational limits in all those cases, but the scalar tilt $n_{s}$ is outside the $3 \sigma$ limit when $\Delta N_{2}>17$, assuming the standard reheating scenario.

PBH masses and their density fraction The mass of a PBH created by late-inflationary overdensities was estimated in Ref. [14] as follows:

$$
M_{\mathrm{PBH}} \simeq \frac{M_{\mathrm{Pl}}^{2}}{H\left(t_{*}\right)} \exp \left[2\left(N_{\mathrm{end}}-N_{*}\right)+\int_{t_{*}}^{t_{\text {exit }}} \epsilon(t) H(t) d t\right],
$$

where $t_{*}$ is the time when the first (slow-roll) stage ends, whereas $t_{\text {exit }}$ is the time when the CMB pivot scale $k=$ $0.05 \mathrm{Mpc}^{-1}$ exits the horizon. The formula is independent of the period between $t_{*}$ and the time of PBHs formation during the radiation-dominated era.

We estimate the values of $M_{\mathrm{PBH}}$ for various values of $\Delta N_{2}$ by using Eq. (63). The results are shown in Table 3 together with the corresponding values of the spectral index. Our estimates are universal across the values of $\gamma=$ $0.1,1,10,100,1000$. PBHs with masses smaller than $10^{16} \mathrm{~g}$ would have already evaporated by now via Hawking radiation. Thus, on one hand, we need $\Delta N_{2}>17$. On the other hand, the lower $3 \sigma$ limit on the spectral index, $n_{s} \approx 0.946$ [21], requires $\Delta N_{2} \leq 17$. Hence, the $\gamma$ extension alone is apparently ruled out as a model of PBH DM when we assume the standard reheating scenario and demand PBHs formation during the radiation era. Therefore, either we need yet another extension of our ansatz in modified supergravity or we have to assume some alternative (nonstandard) cosmological scenarios.

As regards the constraints on $\gamma$, the power spectrum in Fig. 8 tells us that it is sufficient to have $\gamma \geq \mathcal{O}(1)$ in order to produce the required enhancement in the spectrum.

We also estimate the PBHs density fraction by using Press-Schechter formalism [52]. The useful formulae include 

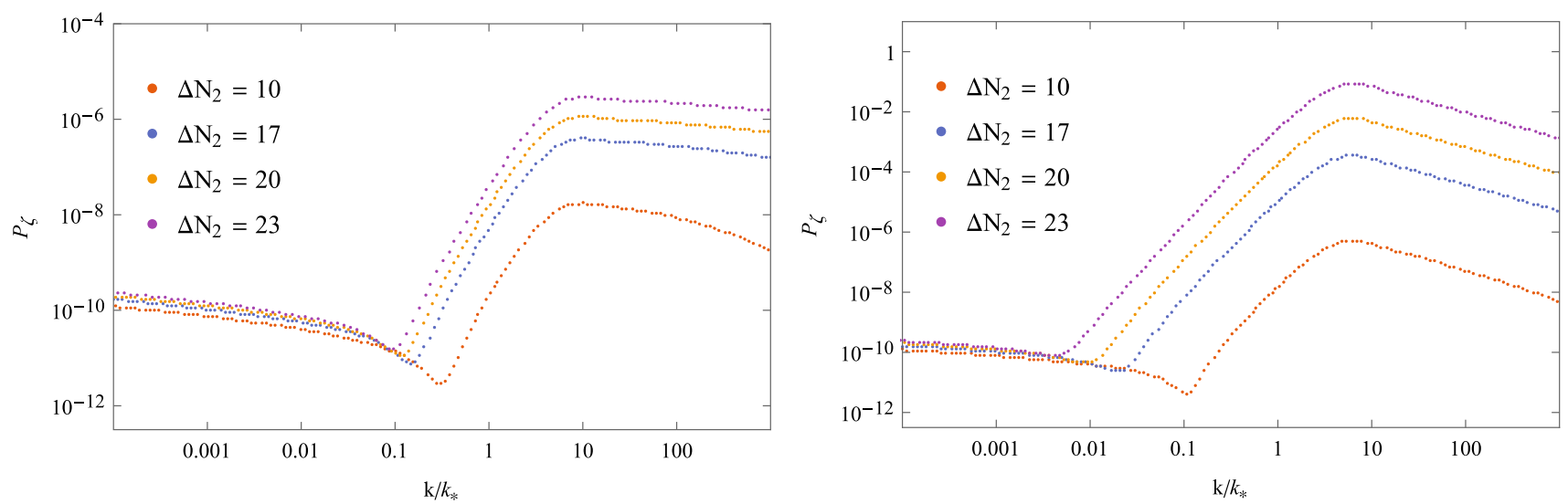

Fig. 8 The power spectrum at $\gamma=0.1$ (on the left side) and $\gamma=1$ (on the right side) for $\Delta N_{2}=10,17,20,23$

Table 2 The approximate values of $n_{s}$ and $r$ for various choices of $\Delta N_{2}$, obtained by tuning the parameter $\zeta$ around its inflection point value

\begin{tabular}{lllll}
\hline$\Delta N_{2}$ & 10 & 17 & 20 & 23 \\
\hline$n_{s}$ & 0.955 & 0.946 & 0.942 & 0.936 \\
$r_{\max }$ & 0.006 & 0.008 & 0.009 & 0.011 \\
\hline
\end{tabular}

the PBH mass $\tilde{M}_{\mathrm{PBH}}(k)$, the production rate $\beta_{f}(k)$, and the density contrast $\sigma(k)$ coarse-grained over $k$ as follows (see e.g., Refs. $[53,54]$ and references therein):

$\tilde{M}_{\mathrm{PBH}} \simeq 10^{20}\left(\frac{7 \times 10^{12}}{k \mathrm{Mpc}}\right)^{2} \mathrm{~g}, \quad \beta_{f}(k) \simeq \frac{\sigma(k)}{\sqrt{2 \pi} \delta_{c}} e^{-\frac{\delta_{c}^{2}}{2 \sigma^{2}(k)}}$,

$\sigma^{2}(k)=\frac{16}{81} \int \frac{d q}{q}\left(\frac{q}{k}\right)^{4} e^{-q^{2} / k^{2}} P_{\zeta}(q)$,

respectively, where we have chosen the Gaussian window function for the density contrast and have introduced $\delta_{c}$ as a constant representing the density threshold for $\mathrm{PBH}$ formation, which is usually estimated as $\delta_{c} \approx 1 / 3$ [55] for simplicity (its more precise value depends upon details of the power spectrum). In terms of the above functions, the PBH-to-DM density fraction can be estimated as follows [53,54]:

$\frac{\Omega_{\mathrm{PBH}}(k)}{\Omega_{\mathrm{DM}}} \equiv f(k) \simeq \frac{1.2 \times 10^{24} \beta_{f}(k)}{\sqrt{\tilde{M}_{\mathrm{PBH}}(k) \mathrm{g}^{-1}}}$,

where the numerical factor is computed for the Minimal Supersymmetric Standard Model degrees of freedom (in the case of the Standard Model it would be approximately $\left.1.4 \times 10^{24}\right)$.

In order to numerically evaluate the functions (65) and (66), we need to normalize the values of $k$ in terms of the observable scales today. As we already mentioned above, we use the scale $k=0.05 \mathrm{Mpc}^{-1}$ leaving the horizon $54 \mathrm{e}$-folds
Table 3 The PBH masses estimated by Eq. (63) for the $\gamma$-extension with the corresponding (approximate) values of the spectral index. In the Solar mass units, $1 \mathrm{~g} \approx 5.03 \times 10^{-34} M_{\odot}$

\begin{tabular}{lllll}
\hline$\Delta N_{2}$ & 10 & 17 & 20 & 23 \\
\hline$M_{\mathrm{PBH}}, \mathrm{g}$ & $10^{9}$ & $10^{15}$ & $10^{17}$ & $10^{20}$ \\
$n_{s}$ & 0.955 & 0.946 & 0.942 & 0.936 \\
\hline
\end{tabular}

before the end of inflation. To find a specific example, we search for a set of the parameters that can lead to a substantial PBH density with the critical density in the range $1 / 3 \lesssim \delta_{c} \lesssim$ $2 / 3$. We get such an example with $\gamma=1.5, \Delta N_{2}=20$, and $\delta_{c}=0.4$ (this leads to $n_{s} \approx 0.942$ as can be seen in Table 3). The resulting PBH fraction is shown in Fig. 9 on the background of the observational constraints of Ref. [56] (see also Ref. [57]). According to Fig. 9, our peak is located at the edge of the lowest-mass window, between $10^{17}$ and $10^{18} \mathrm{~g}$. The constraints of Fig. 9 are imposed by assuming a monochromatic $\mathrm{PBH}$ mass spectrum. In our case the mass distribution is narrow, albeit is not strictly monochromatic.

The total PBH-to-DM density fraction, given by

$f_{\text {tot }}=\int d\left(\log \tilde{M}_{\mathrm{PBH}}\right) f\left(\tilde{M}_{\mathrm{PBH}}\right)$,

is estimated for Fig. 9 as $f_{\text {tot }} \approx 1$, i.e. PBHs can constitute the whole DM in that case.

\subsection{The $\delta$-extension}

Having established that the PBH DM scenario in the $\gamma$ extension is in conflict with the CMB constraint, the next possibility is to study the $\delta$-extension. In this subsection, we take $\gamma=0$ for simplicity and take $\delta \neq 0$ in Eqs. (53) and (54). ${ }^{8}$ It breaks the R-symmetry and the reflection symme-

\footnotetext{
8 A model similar to our $\delta$-extension was considered in Ref. [58] in relation to spontaneous supersymmetry breaking after inflation. The
} 
Fig. 9 The PBHs fraction obtained with the parameters $\gamma=1.5, \Delta N_{2}=20$, and $\delta_{c}=0.4$ (black curve). The shaded regions represent the observational constraints of Ref. [56]: from evaporation (red), lensing (blue), gravitational waves (gray), various dynamical effects (green), accretion (light blue), large-scale structure (pink), and CMB distortions (orange)
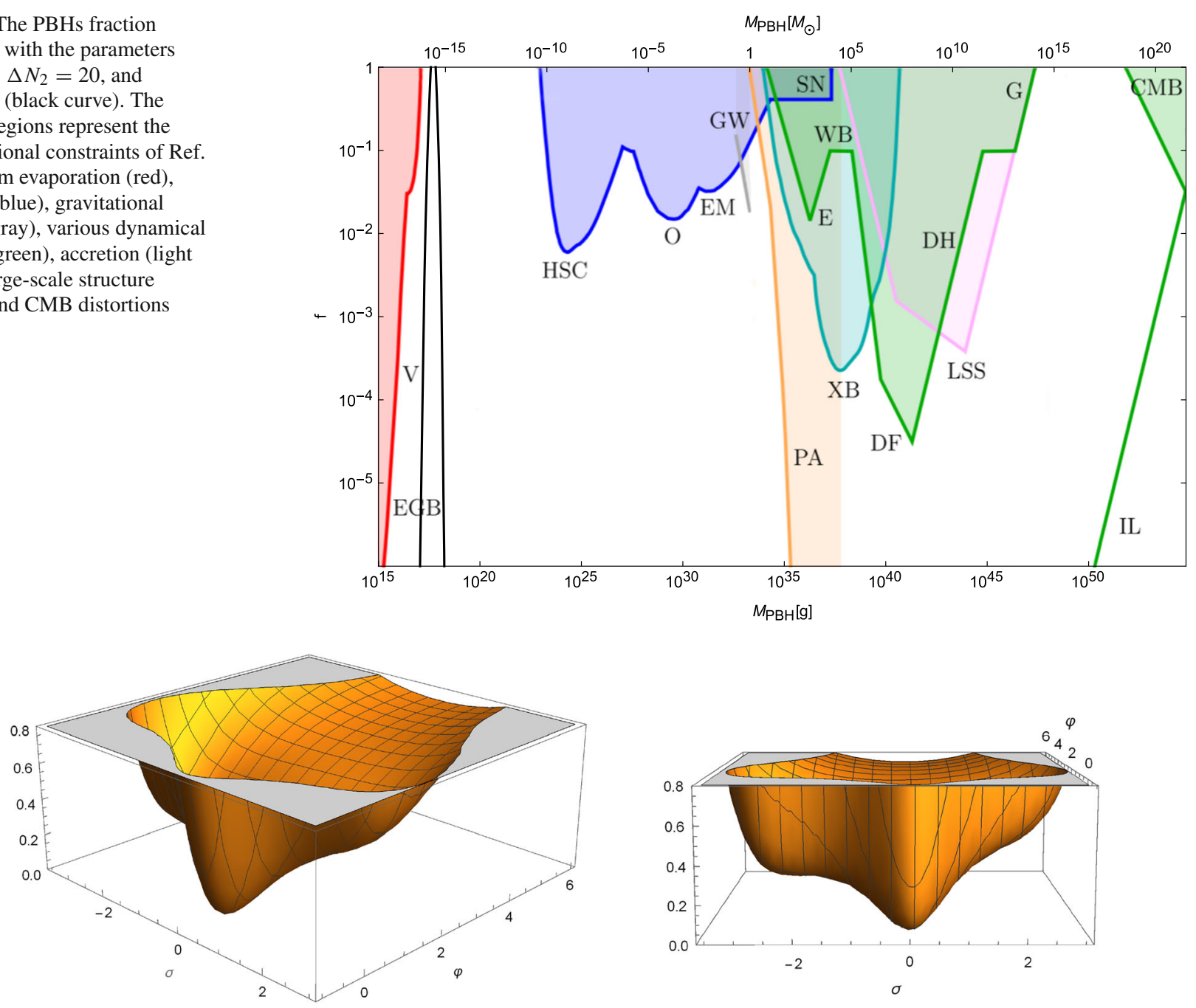

Fig. 10 The scalar potential in the Lagrangian (55) for $\gamma=0, \delta=0.1$ and $\zeta=0.033407$

try $\sigma \rightarrow-\sigma$ of the potential (see Fig. 10), and generates $\theta$-dependent terms in the potential (we remind that $\theta$ is the angular component of the complex scalar $X \equiv \mathcal{R} \mid$ ). Those terms can be obtained by replacing $\delta \rightarrow \delta \cos \theta$ in Eq. (56). We assume that the angular scalar $\theta$ is stabilized during inflation. Then its VEV $\langle\theta\rangle$ can be absorbed into a redefinition of $\delta$. According to Eq. (56), the $\theta$ terms are multiplied by the factors of $\sigma$, so that they vanish when $\sigma=0$, including the Minkowski minimum. A stabilization of $\theta$ requires additional tools that we leave to future studies.

As far as the shape of the potential is concerned, for any non-zero $\delta$ there is a value of $\zeta$ that leads to an inflection point:

Footnote 8 continued

difference between our model and that of Ref. [58] is in the scalar potential: the potential of Ref. [58] has an additional (Minkowski) minimum (away from $\sigma=0$ ) that breaks both supersymmetry and R-symmetry. In our case, we have a single, SUSY-preserving Minkowski minimum (at $\sigma=0$ ) and an inflection point away from $\sigma=0$, in order to achieve an ultra-slow-roll stage. for a positive $\delta$ the inflection point is at $\sigma=-\left|\sigma_{\text {inflec }}\right|$ (as in our example of Fig. 10), and for a negative $\delta$ the inflection point is at $\sigma=+\left|\sigma_{\text {inflec }}\right|$.

In contrast to the $\gamma$-extension, here we have a single valley for large positive $\varphi$ and $\sigma=0$, so that in this limit the model reduces to a single-field Starobinsky model. As one approaches $\varphi=0$, the potential inclines towards the (near-)inflection point which could, in principle, guide the inflationary trajectory towards passing through the (near)inflection point before falling to the Minkowski minimum at $\varphi=\sigma=0$.

Let us consider, for example, the parameter values $\delta=$ 0.1 and $\zeta=0.033407$ ( $\zeta$ is chosen to get $\Delta N_{2}=10$ ). After solving the corresponding field equations, we show the time dependence of $\varphi, \sigma, \tilde{H}, N, \epsilon$ and $\eta_{\Sigma \Sigma}$ in Fig. 11. The near-inflection point divides inflation into two stages with $\Delta N_{1}=50$ (slow-roll) and $\Delta N_{2}=10$ (ultra-slow-roll). We set initial velocities to zero, with $\varphi(0)=6$ and $\sigma(0)=0.05$. 


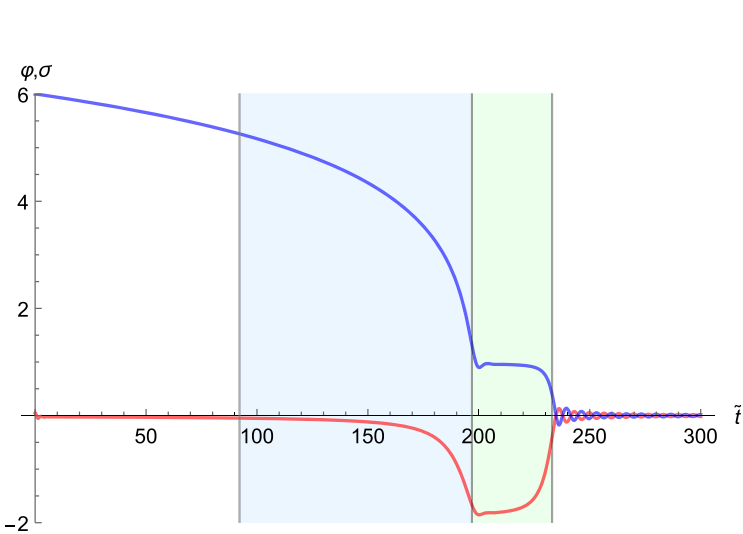

(a)

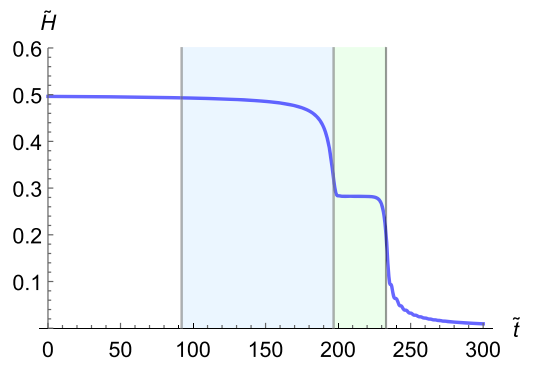

(c)

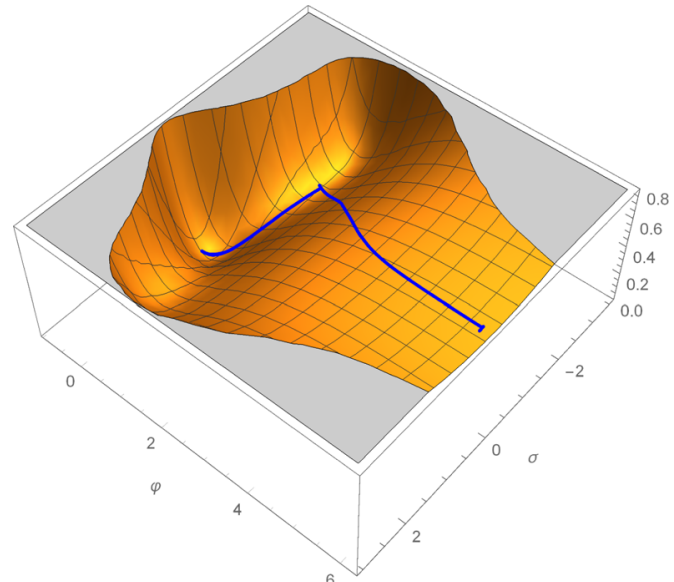

(b)

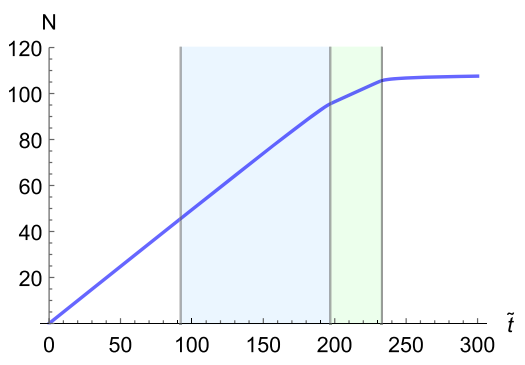

(d)

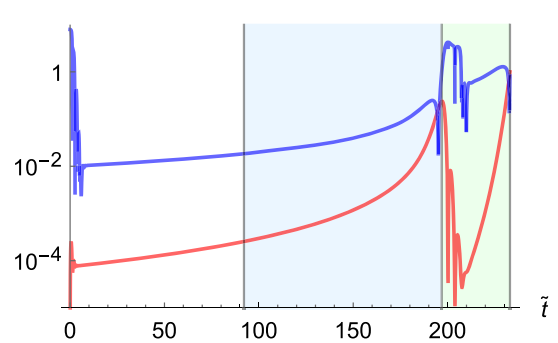

(e)
Fig. 11 a The solution to the field equations (57) and (58) with the initial conditions $\varphi(0)=6$ and $\sigma(0)=0.05$, the vanishing initial velocities, and the parameter choice $\gamma=0, \delta=0.1$ and $\zeta=0.033407$. b The trajectory of the solution ( $\varphi-$ blue, $\sigma-$ red). c The corresponding Hubble function. d The number of e-folds. e The slow-roll parameters $\epsilon$ (red) and $\eta_{\Sigma \Sigma}$ (blue)
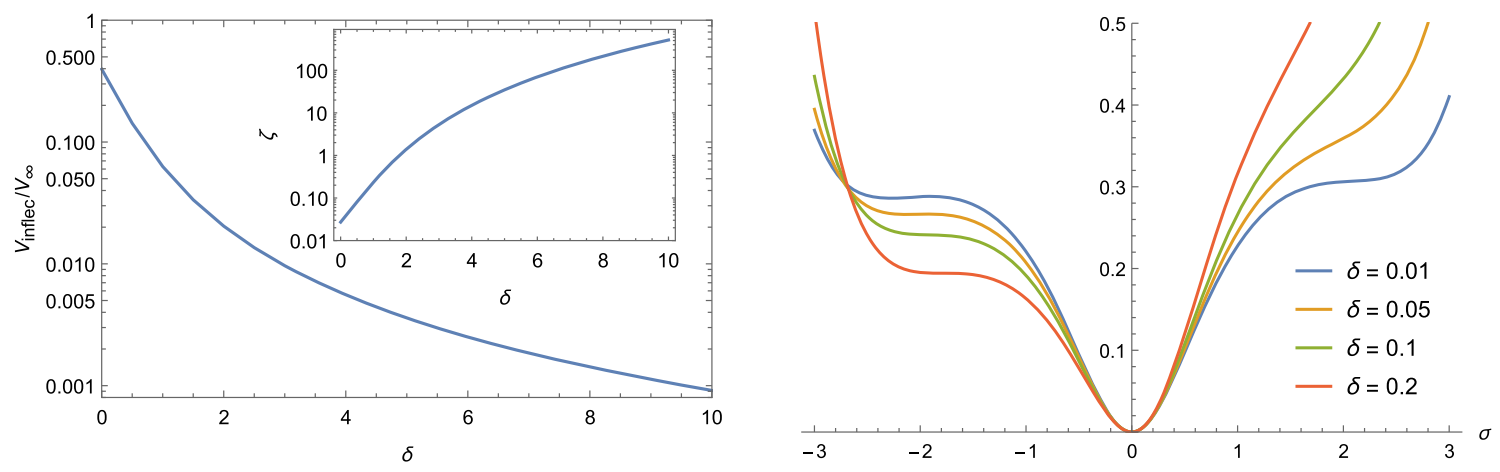

Fig. 12 The left side: the ratio $V_{\text {inflec }} / V_{\infty}$ and $\zeta$ as the functions of $\delta$ according to Eq. (62). The right side: the profile of the potential $V / M^{2}$ with a single inflection point, when $\varphi$ is at its local minimum

Similarly to the $\gamma$-extension, the inflationary trajectory is stable against variations of the initial conditions, as long as they are not very large.

The parameter space When demanding the presence of a (near-)inflection point, the parameters must satisfy Eq. (62). The plot of $V_{\text {inflec. }} / V_{\infty}\left(V_{\infty}\right.$ is taken for $\varphi \gg 1$ and $\left.\sigma=0\right)$ versus $\delta$, and the solution $\zeta(\delta)$ to Eq. (62), are displayed on the left side of Fig. 12. On the right side of Fig. 12 we show the profile of the potential with $\varphi$ at its local minimum satisfying $\partial_{\varphi} V=0$, for several choices of $\delta$. In particular, our plot shows, when $\delta \rightarrow 0$, the inclination of the potential towards the inflection point becomes smaller until it vanishes when $\delta=0$ (in such case the potential coincides with the one in Fig. 1b). Therefore, when $\delta$ is very small, the inclination of the potential becomes insufficient for guiding the inflationary trajectory through the inflection point. Instead, the trajectory tends to the $\sigma=0$ path (when $\delta=0$, the trajectory exactly follows the $\sigma=0$ path). 


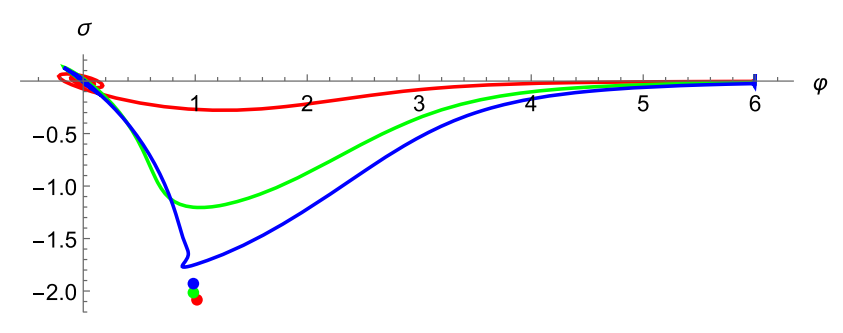

Fig. 13 The inflationary trajectories and the corresponding inflection points marked by the colored points. The parameters are $\delta=0.01$ (red), $\delta=0.05$ (green) and $\delta=0.1$ (blue)

We plot the inflationary trajectories in the $\varphi-\sigma$ plane for various choices of $\delta$ (with $\zeta$ being fixed by requiring the existence of an inflection point) in Fig. 13. The colored spots represent the inflection points. As can be seen in Fig. 13 for $\delta=0.01$ and $\delta=0.05$, the trajectory misses the corresponding inflection point by a large margin and, therefore, avoids the USR regime. On the other hand, when $\delta=0.1$, the trajectory stops near the inflection point and then oscillates a few times before going to the minimum at $\varphi=\sigma=0$. This indicates the possibility of an USR stage, and it happens in Fig. 11 for this parameter choice indeed.

The scalar power spectrum at fixed $\Delta N_{2}$ and $\delta \gtrsim 0.1$ Let us fix $\Delta N_{2}=10$, and consider the power spectrum for several values of $\delta$. We find that the spectrum has a non-trivial dependence on $\delta$, see Fig. 14. In the left plot, $\delta$ is varied from 0.1 to 0.2 , and we observe the spectrum enhancement to become smaller as $\delta$ grows. In the right plot, once $\delta$ reaches 0.2 , the enhancement starts growing with $\delta$ and develops a sharper peak.

As regards larger values of $\delta$, our numerical results show, when $\delta \gtrsim 0.6$, it becomes increasingly more difficult to maintain the USR stage and to achieve $\Delta N_{2}>10$, in particular. It may be due to the need of an extreme fine-tuning of the parameter $\zeta$ when $\delta$ is large.

Changing $\Delta N_{2}$ Amongst the values of $\delta$ studied above, let us pick up those with the highest power spectrum peaks, namely, $\delta=0.1$ and $\delta=0.6$, and plot the spectrum for $\Delta N_{2}=10,17,20,23$. The results are displayed in Fig. 15 with the plots on the left side and the right side corresponding to $\delta=0.1$ and 0.6 , respectively. As expected, the enhancement becomes larger with increasing $\triangle N_{2}$.

PBH masses and their density fraction To be specific, let us consider two different examples: a smooth peak for $\delta \sim$ 0.1 , and a sharp peak for $\delta \sim 0.6$, in the power spectrum. Requiring the total PBH density fraction $f_{\text {tot }} \approx 1$ and the corresponding density threshold in the region $1 / 3 \leq \delta_{c} \leq$ $2 / 3$, we find $\delta=0.09$ and $\delta=0.61$ are suitable for efficient generation of PBHs.

We estimate the PBH masses by using Eq. (63) and summarize our results in Table 4. We find when $\delta=0.09$, the $3 \sigma$ value of $n_{s} \gtrsim 0.946$ requires $\Delta N_{2} \lesssim 19$. When $\delta=0.61$, it requires $\Delta N_{2} \lesssim 20$. In the examples of $\delta=0.09$ and $\delta=0.61$ we take the upper limits $\Delta N_{2}=19$ and $\Delta N_{2}=20$, respectively, and compute the $\mathrm{PBH}$ density fraction from Eq. (66). The results are shown in Fig. 16 where the observational constraints are included for the reference purposes only, as they assume the monochromatic PBH mass function. The density functions of Fig. 16 peak at $M_{\mathrm{PBH}} \sim 10^{20} \mathrm{~g}$ and $M_{\mathrm{PBH}} \sim 10^{18} \mathrm{~g}$ when $\delta=0.09$ and $\delta=0.61$, respectively. ${ }^{9}$

\section{Conclusions and comments}

In this paper, we analyze several supergravity extensions of the Starobinsky inflationary model. We explore possibilities of PBHs genesis that could account for part of Cold Dark Matter. We find that PBHs generation can be efficiently catalyzed by primordial perturbations sourced by the Starobinsky scalaron coupled to a new supersymmetric "modulus" (scalar) field.

Let us summarize our strategy.

We rely on theoretical considerations before comparing them with cosmological observations, as a top-down approach. As our starting point, we adopt the Starobinsky inflationary model serving as the theoretical tool and pointing out the need of modified gravity in a more fundamental approach, i.e. beyond considering the Starobinsky model as merely the best phenomenological fit to CMB observations. We extend the modified $\left(R+\zeta R^{2}\right)$ gravity to the modified supergravity, where the latter is considered as the candidate (or as the approximation) of a more fundamental theory of quantum gravity. Amongst the theoretical advantages of modified supergravity are (i) the use of supergravitational couplings only, (ii) predicted new physical degrees of freedom, and (iii) its formal equivalence to the standard (matter-coupled) supergravities. However, unlike the standard supergravities coupled to matter, modified supergravity can be limited to the supergravity fields alone, where the new physical scalar naturally accompanies the inflaton (scalaron), together with metric and gravitino. It happens because modified supergravity is a higher-derivative theory, so that the "auxiliary" scalar of the standard (off-shell) supergravity multiplet becomes dynamical. We find that modified supergravity naturally leads to the two-field inflationary models with restricted couplings and a small number of free parameters. Therefore, local supersymmetry has predictive power for phenomenology of the early universe cosmology via the double-inflation scenario. Indeed, the second field coupled to the Starobinsky scalaron is not introduced ad hoc but is predicted by the supergravity extension of the

\footnotetext{
${ }^{9}$ The peak in the PBH density of the case $\delta=0.09$ can be seen to deviate from the prediction of Eq. (63) shown in Table 4, likely due to the relatively broad nature of the peak.
} 
Starobinsky model. Our strategy is to use those models for a viable description of Starobinsky inflation together with the $\mathrm{PBH}$ production after inflation. Cosmological inflation and the PBH production can be considered as probes of supergravity for its use as a more fundamental approach, and vice versa: modified supergravity provides a theoretical input for the discrimination of phenomenological models of inflation and PBHs.

We summarize our main results as follows.

A generic modified supergravity Lagrangian in the manifestly supersymmetric form (with all couplings included) is given by Eq. (1). After (Taylor) expanding its potentials $N$ and $\mathcal{F}$ in powers of the scalar curvature superfield $\mathcal{R}$ and keeping only the leading terms (needed for minimal embed- ding of $R+\zeta R^{2}$ gravity), we arrive at our basic model defined by Eq. (2), whose relevant bosonic terms (in Jordan frame) are given by Eqs. (3) and (4). As the next step, we perform the duality transformation of the derived bosonic terms to Einstein frame, and arrive at the two-scalar NLSM minimally coupled to gravity with the derived NLSM metric and the scalar potential, given by Eqs. (14) and (15). We also provide the manifestly supersymmetric (complete) duality transformation in terms of the superfields, and compute the corresponding Kähler potential and the superpotential, given by Eqs. (20) and (21) in the case of the basic model as an example. Then, we study the critical points (vacua) of the derived scalar potentials and the inflationary dynamics of two scalars in the context of two-field inflation, and we
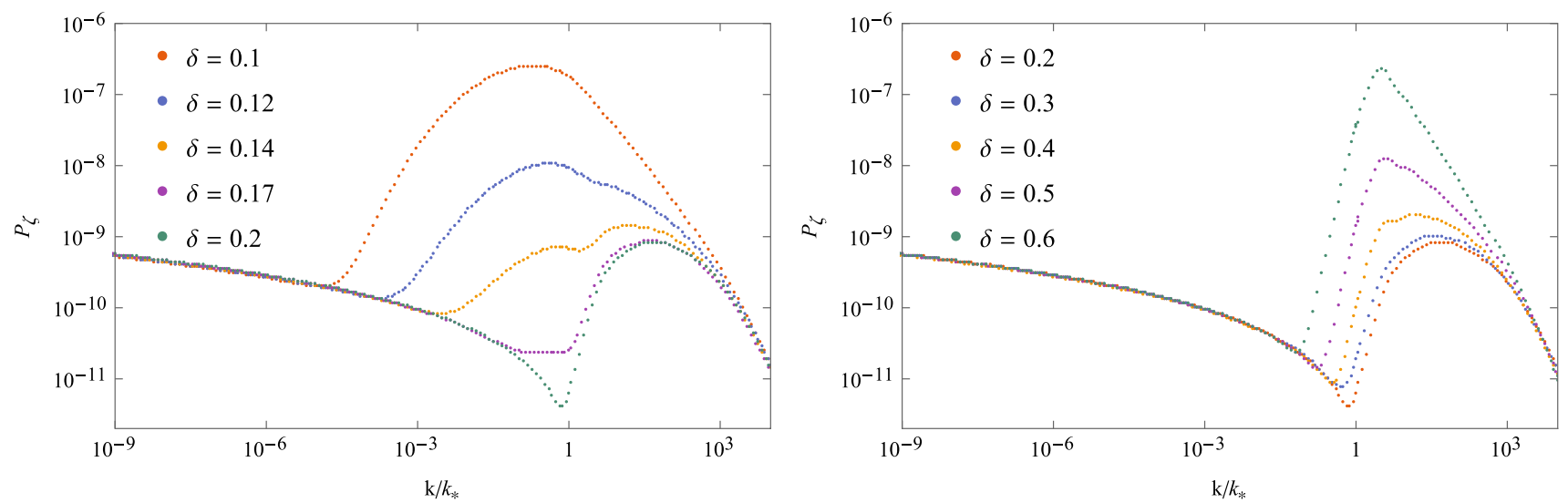

Fig. 14 The power spectrum for various values of $\delta$ from 0.1 to 0.2 (on the left side), and from 0.2 to 0.6 (on the right side). The pivot scale is $k_{*}=k_{\Delta N_{2}}$ with $\Delta N_{2}=10$
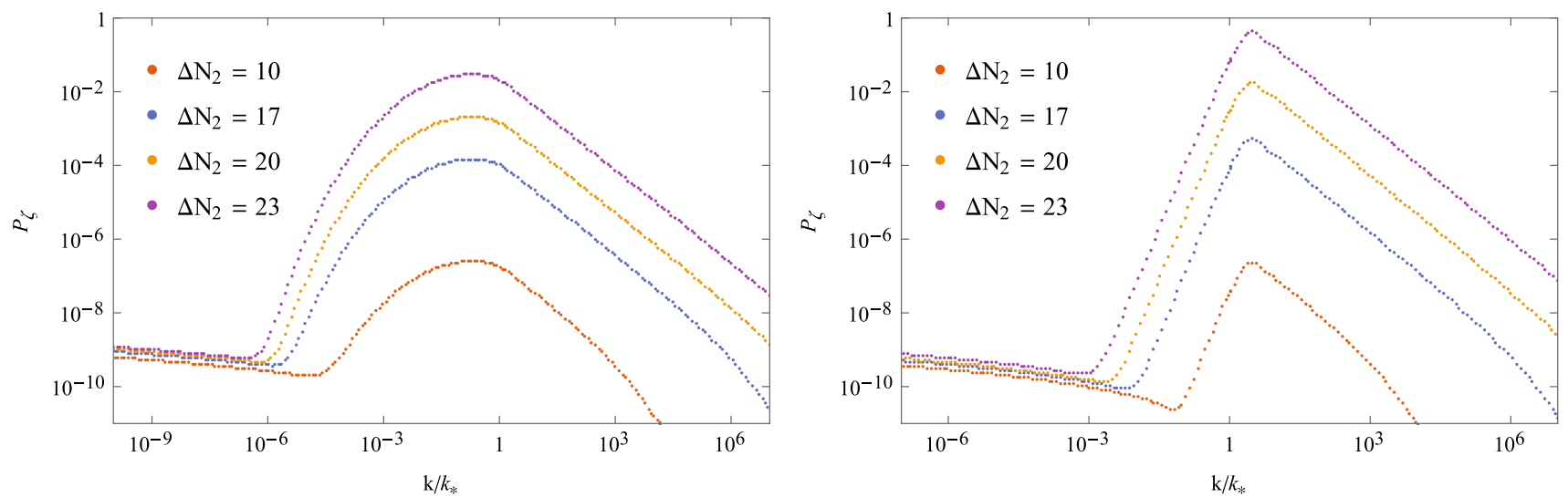

Fig. 15 The power spectrum for $\delta=0.1$ (left) and $\delta=0.6$ (right) around the pivot scale $k_{*}=k_{\Delta N_{2}}$ when changing $\Delta N_{2}$

Table 4 The PBH masses estimated from Eq. (63) for $\delta=0.09$ and $\delta=0.61$, with the corresponding values of $n_{s}$ and $r_{\max }$

\begin{tabular}{|c|c|c|c|c|c|c|c|c|}
\hline \multirow[t]{2}{*}{$\Delta N_{2}$} & \multicolumn{4}{|c|}{$\delta=0.09$} & \multicolumn{4}{|c|}{$\delta=0.61$} \\
\hline & 10 & 17 & 20 & 23 & 10 & 17 & 20 & 23 \\
\hline$M_{\mathrm{PBH}}, \mathrm{g}$ & $10^{9}$ & $10^{15}$ & $10^{18}$ & $10^{20}$ & $10^{9}$ & $10^{15}$ & $10^{18}$ & $10^{20}$ \\
\hline$n_{s}$ & 0.9566 & 0.9486 & 0.9443 & 0.9390 & 0.9581 & 0.9504 & 0.9461 & 0.9409 \\
\hline$r_{\max }$ & 0.005 & 0.007 & 0.008 & 0.010 & 0.004 & 0.006 & 0.007 & 0.008 \\
\hline
\end{tabular}


Fig. 16 The PBH fraction in the two working examples of the $\delta$-type extension, including $f_{\text {tot }} \approx 1$. The solid black curve corresponds to $\delta=0.09$, $\Delta N_{2}=19$, and $\delta_{c}=0.47$; the dashed black curve corresponds to $\delta=0.61, \Delta N_{2}=20$, and $\delta_{c}=0.4$. The observational constraints are taken from Ref. [56]

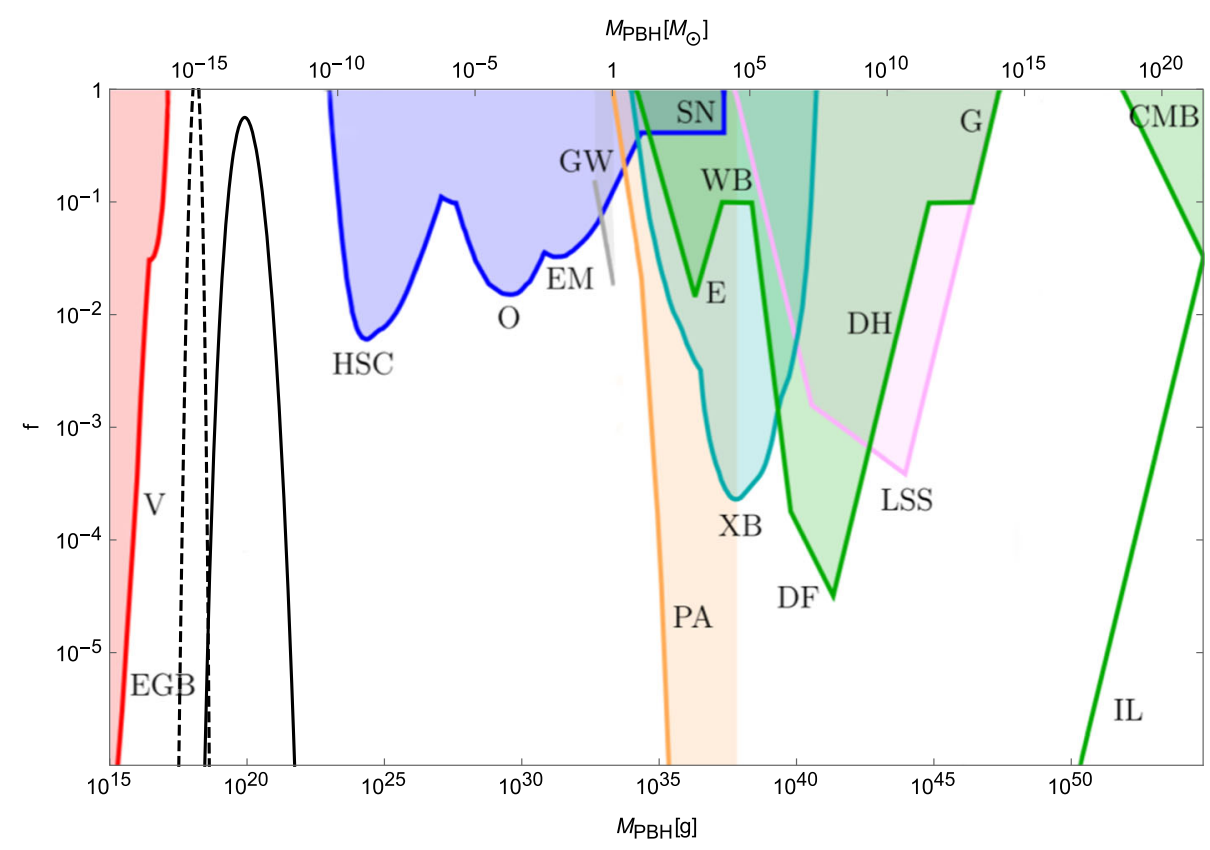

find consistency of the basic model with CMB observations. However, we also observe that such a scenario can work only with an extreme fine-tuning of initial conditions for efficient formation of PBHs.

To overcome that problem, we add the next (subleading) terms to our basic model within the same modified supergravity master Lagrangian (1). There are two such terms, see Eqs. (53) and (54), so we study them separately. We numerically compute the power spectra, estimate PBH masses and their density fraction, in both cases. We find that any of the extended models can simultaneously describe viable (Starobinsky-type) inflation and the PBH production after inflation, with limited fine-tuning of the parameters, exhibiting an attractor-type behavior. Actually, the PBH production is less sensitive to changes of the parameter $\gamma$ in the $\gamma$-extension of the $N$-potential. Next, we confront our theoretical predictions for PBHs (as part of DM) with current observations in Figs. 9 and 16; in the cases of the $\gamma$ - and $\delta$ extensions, respectively. When assuming the standard reheating temperature of $10^{9} \mathrm{GeV}$ with PBHs formation during the radiation era, the $\gamma$-model is apparently ruled out by the $\mathrm{CMB}$ constraints because it predicts $n_{s}$ outside the $3 \sigma$ limit. It motivates us to consider the $\delta$-extension that predicts a larger $n_{s}$ within the $\mathrm{CMB}$ constraint. It is, therefore, quite possible that having both the $\gamma$ - and $\delta$-terms (and, perhaps, even higher order terms) in the Lagrangian will render our supergravity model more flexible in accommodating the PBHs DM.

Of course, modified supergravity does not pretend on the status of an ultimate fundamental theory. However, there are indications that it may be embedded into superstrings considered as an ultra-violet complete theory of quantum gravity. Here it is worthwhile to mention that (i) modified supergravity always leads to the no-scale Kähler potential (20) that often arises in superstring compactifications (see e.g., Ref. [59]), and (ii) there is a possibility of interpreting (some) modified supergravity theories as the D3-brane worldvolume theories in type II superstrings $[60,61]$. Thus, the exploration of cosmological predictions from modified supergravity provides a remarkable bridge between quantum gravity on one side and phenomenology of inflation and PBHs on the other side.

PBH formation necessarily leads to gravitational waves (GWs) because large scalar overdensities act as a source for stochastic GWs background. Frequencies of those GWs can be directly related to expected PBHs masses and duration of the second stage of inflation [62]. Those GWs may be detected in the future ground-based experiments, such as the Einstein telescope [63] and the global network of GWs interferometers including advanced LIGO, Virgo and KAGRA [64], as well as in the space-based GWs interferometers such as LISA [65], TAIJI (old ALIA) [66], TianQin [67] and DECIGO [68].

Acknowledgements The authors are grateful to Fotis Farakos, Anirudh Gundhi, Florian Kühnel, Hayato Motohashi, Misao Sasaki, Alexei Starobinsky, Christian Steinwachs, Spyros Sypsas and Ying-li Zhang for discussions and comments. Y.A. is supported by the CUniverse research promotion project of Chulalongkorn University in Bangkok, Thailand, under the grant reference CUAASC, and by the Ministry of Education and Science of the Republic of Kazakhstan under the grant reference BR05236730. S.V.K. is supported by Tokyo Metropolitan University, the World Premier International Research Center Initiative (WPI), MEXT, Japan, and the Competitiveness Enhancement Program of Tomsk Polytechnic University in Russia.

Data Availability Statement This manuscript has no associated data or the data will not be deposited. [Authors' comment: All the relevant data is included in the provided figures, as well as in the cited articles.] 
Open Access This article is licensed under a Creative Commons Attribution 4.0 International License, which permits use, sharing, adaptation, distribution and reproduction in any medium or format, as long as you give appropriate credit to the original author(s) and the source, provide a link to the Creative Commons licence, and indicate if changes were made. The images or other third party material in this article are included in the article's Creative Commons licence, unless indicated otherwise in a credit line to the material. If material is not included in the article's Creative Commons licence and your intended use is not permitted by statutory regulation or exceeds the permitted use, you will need to obtain permission directly from the copyright holder. To view a copy of this licence, visit http://creativecomm ons.org/licenses/by/4.0/.

Funded by SCOAP ${ }^{3}$.

\section{Appendix A: Supergravity in curved superspace}

We follow the notation and conventions of Ref. [39] with a few obvious modifications. A standard superspace Lagrangian of chiral superfields $\Phi^{i}$ coupled to supergravity reads $\left(M_{\mathrm{Pl}}=1\right)$

$\mathcal{L}=\int d^{2} \Theta 2 \mathcal{E}\left[\frac{3}{8}\left(\overline{\mathcal{D}}^{2}-8 \mathcal{R}\right) e^{-K\left(\Phi^{i}, \bar{\Phi}^{i}\right) / 3}+W\left(\Phi^{i}\right)\right]+$ h.c.,

where $\mathcal{E}$ is the chiral density superfield, $\mathcal{R}$ is the chiral curvature superfield, $\mathcal{D}_{\alpha}, \overline{\mathcal{D}}_{\dot{\alpha}}$ are the superspace covariant derivatives with $\mathcal{D}^{2} \equiv \mathcal{D}^{\alpha} \mathcal{D}_{\alpha}$ and $\overline{\mathcal{D}}^{2} \equiv \overline{\mathcal{D}}_{\dot{\alpha}} \overline{\mathcal{D}}^{\dot{\alpha}}$. A (real) Kähler potential $K$ and a (holomorphic) superpotential $W$ are functions of the superfields, as indicated above.

A chiral superfield can be expanded in terms of its field components as

$\Phi=\Phi+\sqrt{2} \Theta \chi+\Theta^{2} F$.

The $\Theta$-expansion of $\mathcal{E}$ and $\mathcal{R}$ is given by

$$
\begin{aligned}
2 \mathcal{E}= & e\left[1+i \Theta \sigma^{m} \bar{\psi}_{m}+\Theta^{2}\left(6 \bar{X}-\bar{\psi}_{m} \bar{\sigma}^{m n} \bar{\psi}_{n}\right)\right] \\
\mathcal{R}= & X+\Theta\left(-\frac{1}{6} \sigma^{m} \bar{\sigma}^{n} \psi_{m n}-i \sigma^{m} \bar{\psi}_{m} X-\frac{i}{6} \psi_{m} b^{m}\right) \\
& +\Theta^{2}\left(-\frac{1}{12} R-\frac{i}{6} \bar{\psi}^{m} \bar{\sigma}^{n} \psi_{m n}-4 X \bar{X}-\frac{1}{18} b_{m} b^{m}\right. \\
& +\frac{i}{6} \nabla_{m} b^{m}+\frac{1}{2} \bar{\psi}_{m} \bar{\psi}^{m} X+\frac{1}{12} \psi_{m} \sigma^{m} \bar{\psi}_{n} b^{n} \\
& \left.-\frac{1}{48} \varepsilon^{a b c d}\left(\bar{\psi}_{a} \bar{\sigma}_{b} \psi_{c d}+\psi_{a} \sigma_{b} \bar{\psi}_{c d}\right)\right),
\end{aligned}
$$

where $e \equiv \operatorname{det}\left(e_{m}^{a}\right)$ and $\psi_{m n} \equiv \tilde{D}_{m} \psi_{n}-\tilde{D}_{n} \psi_{m}$ with the covariant derivative $\tilde{D}_{m} \psi_{n} \equiv \partial_{m} \psi_{n}+\psi_{n} \omega_{m}$. The vector $b_{m}$ and complex scalar $X$ are known in the literature as the old-minimal set of supergravity auxiliary fields. In modified supergravity, those "auxiliary" fields become dynamical (or propagating) because of the presence of higher-derivatives in the Lagrangian (see e.g., Ref. [36] for details). In our notation, the scalar curvature $R$ has the opposite sign compared to that in Wess-Bagger notation [39].

In the standard supergravity, after eliminating the auxiliary fields and going to Einstein frame, the bosonic part of the Lagrangian of matter superfields $\Phi^{i}$ takes the form

$$
\begin{aligned}
e^{-1} \mathcal{L}= & \frac{1}{2} R-K_{i \bar{j}} \partial_{m} \Phi^{i} \partial^{m} \bar{\Phi}^{j} \\
& -e^{K}\left(K^{i \bar{j}} D_{i} W D_{\bar{j}} \bar{W}-3|W|^{2}\right),
\end{aligned}
$$

where $K=K\left(\Phi^{i}, \bar{\Phi}^{i}\right)$ is the Kähler potential, and $W=$ $W\left(\Phi^{i}\right)$ is the superpotential, while the same notation is used for the superfields and their leading field components, together with the standard definitions

$$
K_{i \bar{j}} \equiv \frac{\partial^{2} K}{\partial \Phi^{i} \partial \bar{\Phi}^{j}}, \quad K^{i \bar{j}} \equiv K_{i \bar{j}}^{-1}, \quad D_{i} W \equiv \frac{\partial W}{\partial \Phi^{i}}+W \frac{\partial K}{\partial \Phi^{i}} .
$$

\section{Appendix B: Estimating the transfer functions and the isocurvature fraction}

Let us consider the case of $\gamma=1$ and $\Delta N_{2}=10$ as an example. After computing the transfer functions in Eq. (48) as functions of $t_{2}$, with $t_{1}$ being fixed as the time corresponding to 60 e-folds before the end of inflation (it corresponds to the horizon exit of the largest observable scale of around $k=10^{-4} \mathrm{Mpc}^{-1}$ ), we find the result shown in Fig. 17.

Having determined $T_{\mathrm{RS}}$ and $T_{\mathrm{SS}}$, we compute the isocurvature fraction at the end of inflation, i.e. with $t_{2}=t_{\text {end }}$, and get

$\beta_{\text {iso }}=\frac{T_{\mathrm{SS}}^{2}}{1+T_{\mathrm{SS}}^{2}+T_{\mathrm{RS}}^{2}}=\mathcal{O}\left(e^{-1200}\right)$,

which is truly negligible.

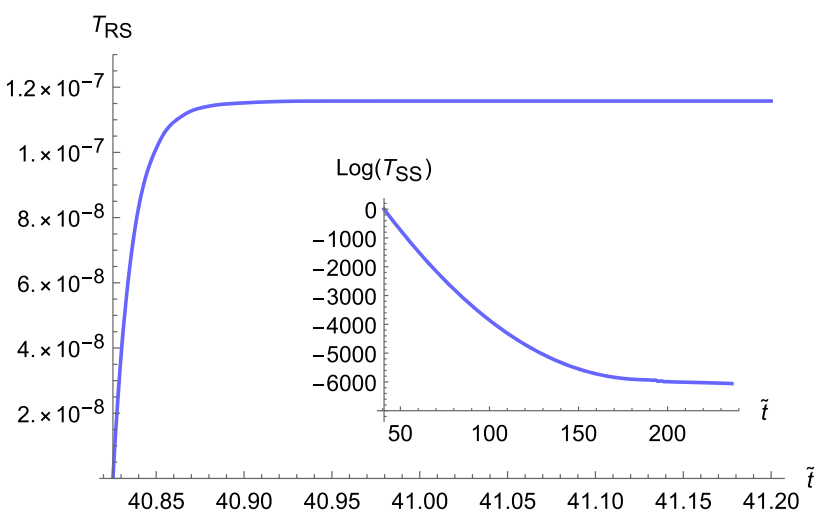

Fig. 17 The time dependence of the transfer functions 


\section{References}

1. I. Novikov, Y. Zeldovic, Cosmology. Ann. Rev. Astron. Astrophys. 5, 627-649 (1967). https://doi.org/10.1146/annurev.aa.05.090167. 003211

2. S. Hawking, Gravitationally collapsed objects of very low mass. Mon. Not. R. Astron. Soc. 152, 75 (1971)

3. M. Khlopov, B. Malomed, I. Zeldovich, Gravitational instability of scalar fields and formation of primordial black holes. Mon. Not. R. Astron. Soc. 215, 575-589 (1985)

4. R. Konoplich, S. Rubin, A. Sakharov, M. Khlopov, Formation of black holes in first-order phase transitions as a cosmological test of symmetry-breaking mechanisms. Phys. Atom. Nucl. 62, 15931600 (1999)

5. M. Khlopov, R. Konoplich, S. Rubin, A. Sakharov, First-order phase transitions as a source of black holes in the early universe. Gravit. Cosmol. 6, 153-156 (2000)

6. A. Addazi, A. Marcianò, R. Pasechnik, Probing trans-electroweak first order phase transitions from gravitational waves. MDPI Phys. 1(1), 92-102 (2019). https://doi.org/10.3390/physics1010010. arXiv:1811.09074 [hep-ph]

7. A. Vilenkin, Y. Levin, A. Gruzinov, Cosmic strings and primordial black holes. JCAP 11, 008 (2018). https://doi.org/10.1088/ 1475-7516/2018/11/008. arXiv:1808.00670 [astro-ph.CO]

8. K.M. Belotsky, V.I. Dokuchaev, Y.N. Eroshenko, E.A. Esipova, M.Y. Khlopov, L.A. Khromykh, A.A. Kirillov, V.V. Nikulin, S.G. Rubin, I.V. Svadkovsky, Clusters of primordial black holes. Eur. Phys. J. C 79(3), 246 (2019). https://doi.org/10.1140/epjc/ s10052-019-6741-4. arXiv:1807.06590 [astro-ph.CO]

9. J. Liu, Z.-K. Guo, R.-G. Cai, Primordial black holes from cosmic domain walls. Phys. Rev. D 101(2), 023513 (2020). https://doi.org/ 10.1103/PhysRevD.101.023513. arXiv:1908.02662 [astro-ph.CO]

10. J.D. Barrow, E.J. Copeland, A.R. Liddle, The cosmology of black hole relics. Phys. Rev. D 46, 645-657 (1992). https://doi.org/10. 1103/PhysRevD.46.645

11. M. Sasaki, T. Suyama, T. Tanaka, S. Yokoyama, Primordial black holes-perspectives in gravitational wave astronomy. Class. Quantum Gravity 35(6), 063001 (2018). https://doi.org/10.1088/ 1361-6382/aaa7b4. arXiv:1801.05235 [astro-ph.CO]

12. S.V. Ketov, M.Y. Khlopov, Cosmological probes of supersymmetric field theory models at superhigh energy scales. Symmetry 11(4), 511 (2019). https://doi.org/10.3390/sym11040511

13. B.J. Carr, Primordial black holes as a probe of cosmology and high energy physics. Lect. Notes Phys. 631, 301-321 (2003). https:// doi.org/10.1007/978-3-540-45230-0_7. arXiv:astro-ph/0310838

14. S. Pi, Y.-L. Zhang, Q.-G. Huang, M. Sasaki, Scalaron from $R^{2}$ gravity as a heavy field. JCAP 05, 042 (2018). https://doi.org/10. 1088/1475-7516/2018/05/042. arXiv:1712.09896 [astro-ph.CO]

15. C. Germani, I. Musco, Abundance of primordial black holes depends on the shape of the inflationary power spectrum. Phys. Rev. Lett. 122(14), 141302 (2019). https://doi.org/10.1103/ PhysRevLett.122.141302. arXiv:1805.04087 [astro-ph.CO]

16. J. Fumagalli, S. Renaux-Petel, J.W. Ronayne, L.T. Witkowski, Turning in the landscape: a new mechanism for generating primordial black holes. arXiv:2004.08369 [hep-th]

17. G.A. Palma, S. Sypsas, C. Zenteno, Seeding primordial black holes in multi-field inflation. arXiv:2004.06106 [astro-ph.CO]

18. R.-G. Cai, Z.-K. Guo, J. Liu, L. Liu, X.-Y. Yang, Primordial black holes and gravitational waves from parametric amplification of curvature perturbations. JCAP 06, 013 (2020). https://doi.org/10. 1017/S174392131900869X. arXiv:1912.10437 [astro-ph.CO]

19. R.-G. Cai, S. Pi, M. Sasaki, Gravitational waves induced by non-Gaussian scalar perturbations. Phys. Rev. Lett. 122(20), 201101 (2019). https://doi.org/10.1103/PhysRevLett.122.201101. arXiv:1810.11000 [astro-ph.CO]
20. C.-M. Deng, Y. Cai, X.-F. Wu, E.-W. Liang, Fast radio bursts from primordial black hole binaries coalescence. Phys. Rev. D 98(12), 123016 (2018). https://doi.org/10.1103/PhysRevD.98. 123016. arXiv:1812.00113 [astro-ph.HE]

21. Planck Collaboration, Y. Akrami et al., Planck 2018 results. X. Constraints on inflation. arXiv:1807.06211 [astro-ph.CO]

22. A.A. Starobinsky, A new type of isotropic cosmological models without singularity. Phys. Lett. B 91(1), 99-102 (1980). https:// doi.org/10.1016/0370-2693(80)90670-X

23. J. Garcia-Bellido, E. Ruiz Morales, Primordial black holes from single field models of inflation. Phys. Dark Univ. 18, 47-54 (2017). https://doi.org/10.1016/j.dark.2017.09.007. arXiv:1702.03901 [astro-ph.CO]

24. H. Motohashi, W. Hu, Primordial black holes and slow-roll violation. Phys. Rev. D 96(6), 063503 (2017). https://doi.org/10.1103/ PhysRevD.96.063503. arXiv:1706.06784 [astro-ph.CO]

25. S. Passaglia, W. Hu, H. Motohashi, Primordial black holes and local non-Gaussianity in canonical inflation. Phys. Rev. D 99(4), 043536 (2019). https://doi.org/10.1103/PhysRevD.99. 043536. arXiv: 1812.08243 [astro-ph.CO]

26. F. Farakos, A. Kehagias, A. Riotto, On the Starobinsky model of inflation from supergravity. Nucl. Phys. B 876, 187-200 (2013). https://doi.org/10.1016/j.nuclphysb.2013. 08.005. arXiv:1307.1137 [hep-th]

27. S. Ferrara, R. Kallosh, A. Linde, M. Porrati, Minimal supergravity models of inflation. Phys. Rev. D 88(8), 085038 (2013). https://doi. org/10.1103/PhysRevD.88.085038. arXiv:1307.7696 [hep-th]

28. Y. Aldabergenov, S.V. Ketov, SUSY breaking after inflation in supergravity with inflaton in a massive vector supermultiplet. Phys. Lett. B 761, 115-118 (2016). https://doi.org/10.1016/j.physletb. 2016.08.016. arXiv:1607.05366 [hep-th]

29. Y. Aldabergenov, S.V. Ketov, Higgs mechanism and cosmological constant in $N=1$ supergravity with inflaton in a vector multiplet. Eur. Phys. J. C 77(4), 233 (2017). https://doi.org/10.1140/epjc/ s10052-017-4807-8. arXiv:1701.08240 [hep-th]

30. A. Addazi, A. Marciano, S.V. Ketov, M.Y. Khlopov, Physics of superheavy dark matter in supergravity. Int. J. Mod. Phys. D 27(06), 1841011 (2018). https://doi.org/10.1142/S0218271818410110

31. J. Garcia-Bellido, A.D. Linde, D. Wands, Density perturbations and black hole formation in hybrid inflation. Phys. Rev. D 54, 6040-6058 (1996). https://doi.org/10.1103/PhysRevD.54. 6040. arXiv:astro-ph/9605094

32. M. Kawasaki, Y. Tada, Can massive primordial black holes be produced in mild waterfall hybrid inflation? JCAP $\mathbf{0 8}$, 041 (2016). https://doi.org/10.1088/1475-7516/2016/08/041. arXiv: 1512.03515 [astro-ph.CO]

33. M. Braglia, D.K. Hazra, F. Finelli, G.F. Smoot, A.A. Starobinsky, Generating PBHs and small-scale GWs in two-field models of inflation. arXiv:2005.02895 [astro-ph.CO]

34. S.V. Ketov, On the equivalence of Starobinsky and Higgs inflationary models in gravity and supergravity. J. Phys. A 53(8), 084001 (2020). https://doi.org/10.1088/1751-8121/ab6a33. arXiv:1911.01008 [hep-th]

35. S.V. Ketov, A.A. Starobinsky, Inflation and non-minimal scalar-curvature coupling in gravity and supergravity. JCAP 08, 022 (2012). https://doi.org/10.1088/1475-7516/2012/08/022. arXiv: 1203.0805 [hep-th]

36. S.V. Ketov, T. Terada, Old-minimal supergravity models of inflation. JHEP 12, 040 (2013). https://doi.org/10.1007/ JHEP12(2013)040. arXiv:1309.7494 [hep-th]

37. A. Addazi, S.V. Ketov, Energy conditions in Starobinsky supergravity. JCAP 03, 061 (2017). https://doi.org/10.1088/1475-7516/ 2017/03/061. arXiv:1701.02450 [hep-th]

38. S. Cecotti, Higher derivative supergravity is equivalent to standard supergravity coupled to matter. 1. Phys. Lett. B 190, 86-92 (1987). https://doi.org/10.1016/0370-2693(87)90844-6 
39. J. Wess, J. Bagger, Supersymmetry and Supergravity (Princeton University Press, Princeton, 1992)

40. R. Kallosh, A. Linde, Superconformal generalizations of the Starobinsky model. JCAP 1306, 028 (2013). https://doi.org/10. 1088/1475-7516/2013/06/028. arXiv:1306.3214 [hep-th]

41. J. Gates, S. James, S.V. Ketov, Superstring-inspired supergravity as the universal source of inflation and quintessence. Phys. Lett. B 674, 59-63 (2009). https://doi.org/10.1016/j.physletb.2009.03. 005. arXiv:0901.2467 [hep-th]

42. K. Schutz, E.I. Sfakianakis, D.I. Kaiser, Multifield inflation after Planck: isocurvature modes from nonminimal couplings. Phys. Rev. D 89(6), 064044 (2014). https://doi.org/10.1103/PhysRevD. 89.064044. arXiv:1310.8285 [astro-ph.CO]

43. A. Gundhi, C.F. Steinwachs, Scalaron-Higgs inflation. Nucl. Phys. B 954, 114989 (2020). https://doi.org/10.1016/j.nuclphysb.2020. 114989. arXiv:1810.10546 [hep-th]

44. D.D. Canko, I.D. Gialamas, G.P. Kodaxis, A simple $F($ mathcal $R, \phi)$ deformation of Starobinsky inflationary model. Eur. Phys. J. C 80(5), 458 (2020). https://doi.org/10.1140/ epjc/s10052-020-8025-4. arXiv:1901.06296 [hep-th]

45. A.R. Liddle, P. Parsons, J.D. Barrow, Formalizing the slow roll approximation in inflation. Phys. Rev. D 50, 72227232 (1994). https://doi.org/10.1103/PhysRevD.50.7222. arXiv:astro-ph/9408015

46. T. Terada, Y. Watanabe, Y. Yamada, J. Yokoyama, Reheating processes after Starobinsky inflation in old-minimal supergravity. JHEP 02, 105 (2015). https://doi.org/10.1007/JHEP02(2015)105. arXiv:1411.6746 [hep-ph]

47. H. Motohashi, A.A. Starobinsky, J. Yokoyama, Inflation with a constant rate of roll. JCAP 09, 018 (2015). https://doi.org/10.1088/ 1475-7516/2015/09/018. arXiv:1411.5021 [astro-ph.CO]

48. D.J. Mulryne, D. Seery, D. Wesley, Moment transport equations for non-Gaussianity. JCAP 01, 024 (2010). https://doi.org/10.1088/ 1475-7516/2010/01/024. arXiv:0909.2256 [astro-ph.CO]

49. D.J. Mulryne, D. Seery, D. Wesley, Moment transport equations for the primordial curvature perturbation. JCAP 04, 030 (2011). https://doi.org/10.1088/1475-7516/2011/04/030. arXiv:1008.3159 [astro-ph.CO]

50. M. Dias, J. Frazer, D. Seery, Computing observables in curved multifield models of inflation - a guide (with code) to the transport method. JCAP 12, 030 (2015). https://doi.org/10.1088/1475-7516/ 2015/12/030. arXiv:1502.03125 [astro-ph.CO]

51. H. Jiang, Y. Wang, Massive fields as systematics for single field inflation. JCAP 06, 038 (2017). https://doi.org/10.1088/ 1475-7516/2017/06/038. arXiv:1703.04477 [astro-ph.CO]

52. W.H. Press, P. Schechter, Formation of galaxies and clusters of galaxies by selfsimilar gravitational condensation. Astrophys. J. 187, 425-438 (1974). https://doi.org/10.1086/152650

53. K. Inomata, M. Kawasaki, K. Mukaida, Y. Tada, T.T. Yanagida, Inflationary primordial black holes as all dark matter. Phys. Rev. D 96(4), 043504 (2017). https://doi.org/10.1103/PhysRevD.96. 043504. arXiv:1701.02544 [astro-ph.CO]

54. K. Inomata, M. Kawasaki, K. Mukaida, T.T. Yanagida, Double inflation as a single origin of primordial black holes for all dark matter and LIGO observations. Phys. Rev. D 97(4), 043514 (2018). https://doi.org/10.1103/PhysRevD.97.043514. arXiv:1711.06129 [astro-ph.CO]
55. B.J. Carr, The Primordial black hole mass spectrum. Astrophys. J. 201, 1-19 (1975). https://doi.org/10.1086/153853

56. B. Carr, F. Kuhnel, Primordial black holes as dark matter: recent developments. arXiv:2006.02838 [astro-ph.CO]

57. B. Carr, K. Kohri, Y. Sendouda, J. Yokoyama, Constraints on primordial black holes. arXiv:2002.12778 [astro-ph.CO]

58. I. Dalianis, F. Farakos, A. Kehagias, A. Riotto, R. von Unge, Supersymmetry breaking and inflation from higher curvature supergravity. JHEP 01, 043 (2015). https://doi.org/10.1007/ JHEP01(2015)043. arXiv:1409.8299 [hep-th]

59. J. Ellis, D.V. Nanopoulos, K.A. Olive, A no-scale supergravity framework for sub-Planckian physics. Phys. Rev. D 89(4), 043502 (2014). https://doi.org/10.1103/PhysRevD.89. 043502. arXiv: 1310.4770 [hep-ph]

60. P. Binetruy, G. Dvali, R. Kallosh, A. Van Proeyen, Fayet-Iliopoulos terms in supergravity and cosmology. Class. Quantum Gravity 21, 3137-3170 (2004). https://doi.org/10.1088/0264-9381/21/13/ 005. arXiv:hep-th/0402046

61. Y. Aldabergenov, S. Aoki, S.V. Ketov, Minimal Starobinsky supergravity coupled to a dilaton-axion superfield. Phys. Rev. D 101(7), 075012 (2020). https://doi.org/10.1103/PhysRevD.101. 075012. arXiv:2001.09574 [hep-th]

62. N. Bartolo, V. De Luca, G. Franciolini, A. Lewis, M. Peloso, A. Riotto, Primordial black hole dark matter: LISA serendipity. Phys. Rev. Lett. 122(21), 211301 (2019). https://doi.org/10.1103/ PhysRevLett.122.211301. arXiv:1810.12218 [astro-ph.CO]

63. E.T. Collaboration, M. Punturo et al., The Einstein Telescope: a third-generation gravitational wave observatory. Class. Quantum Gravity 27(19), 194002 (2010). https://doi.org/10.1088/ 0264-9381/27/19/194002

64. KAGRA, LIGO Scientific, VIRGO Collaboration, B. Abbott et al., Prospects for observing and localizing gravitational-wave transients with advanced LIGO, Advanced Virgo and KAGRA. Living Rev. Relativ. 21(1), 3 (2018). https://doi.org/10.1007/ s41114-018-0012-9. arXiv:1304.0670 [gr-qc]

65. LISA Collaboration, P. Amaro-Seoane et al., Laser interferometer space antenna. arXiv:1702.00786 [astro-ph.IM]

66. X. Gong et al., Descope of the ALIA mission. J. Phys. Conf. Ser. 610(1), 012011 (2015). https://doi.org/10.1088/1742-6596/610/1/ 012011. arXiv:1410.7296 [gr-qc]

67. TianQin Collaboration, J. Luo et al., TianQin: a space-borne gravitational wave detector. Class. Quantum Gravity 33(3), 035010 (2016). https://doi.org/10.1088/0264-9381/33/3/035010. arXiv:1512.02076 [astro-ph.IM]

68. H. Kudoh, A. Taruya, T. Hiramatsu, Y. Himemoto, Detecting a gravitational-wave background with next-generation space interferometers. Phys. Rev. D 73, 064006 (2006). https://doi.org/10. 1103/PhysRevD.73.064006. arXiv:gr-qc/0511145 